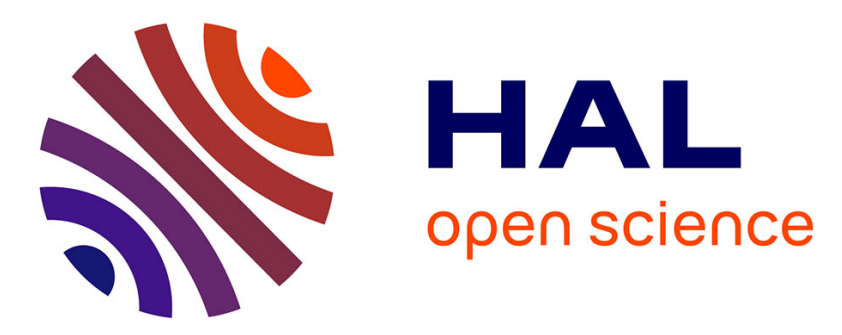

\title{
Comparison of Monte Carlo methods for adjoint neutron transport
}

\author{
V. Vitali, S. Dulla, P. Ravetto, A. Zoia
}

\section{To cite this version:}

V. Vitali, S. Dulla, P. Ravetto, A. Zoia. Comparison of Monte Carlo methods for adjoint neutron transport. The European Physical Journal Plus, 2018, 133 (8), pp.317. 10.1140/epjp/i2018-12132-9 . cea-02339764

\section{HAL Id: cea-02339764 https://hal-cea.archives-ouvertes.fr/cea-02339764}

Submitted on 5 Nov 2019

HAL is a multi-disciplinary open access archive for the deposit and dissemination of scientific research documents, whether they are published or not. The documents may come from teaching and research institutions in France or abroad, or from public or private research centers.
L'archive ouverte pluridisciplinaire HAL, est destinée au dépôt et à la diffusion de documents scientifiques de niveau recherche, publiés ou non, émanant des établissements d'enseignement et de recherche français ou étrangers, des laboratoires publics ou privés. 
EPJ manuscript No.

(will be inserted by the editor)

\title{
Comparison of Monte Carlo methods for adjoint neutron transport
}

\author{
Vito Vitali ${ }^{1}$, Sandra Dulla ${ }^{2}$, Piero Ravetto $^{2}$, and Andrea Zoia ${ }^{1 \mathrm{a}}$ \\ 1 DEN-Service d'études des réacteurs et de mathématiques appliquées (SERMA), CEA, Université Paris-Saclay, F-91191, Gif- \\ sur-Yvette, France \\ 2 Politecnico di Torino, Dipartimento Energia, NEMO Group, Corso Duca degli Abruzzi 24, 10129, Torino, Italy
}

Received: date / Revised version: date

\begin{abstract}
Solving the adjoint linear transport equation by Monte Carlo methods can be convenient for applications emerging in radiation shielding, where the detector is typically small (in terms of probability of detecting a signal). In this work we compare a few stochastic models that can be used in order to formally solve the adjoint transport equation by simulating artificial particles called adjunctons: these models differ in the form of adjuncton cross sections, scattering laws and multiplicities. In view of testing the accuracy and the performances of these schemes, we have selected some benchmark configurations for continuous-energy transport in infinite media, where reference solutions can be established. The role of population control techniques, such as Russian roulette and splitting, is also carefully examined.
\end{abstract}

PACS. 28.41.Ak Theory, design, and computerized simulation - 02.50.Ng Distribution theory and Monte Carlo studies

\section{Introduction}

In the context of radiation transport, Monte Carlo simulation is considered as the golden standard for the computation of physical quantities and is traditionally adopted so as to establish reference values for faster, but approximated, deterministic calculations [1]. Monte Carlo codes are intrinsically based on the simulation of forward random walks, with neutrons flowing from sources and depositing their scores when (and if) they cross or interact with the detectors: as such, they are ideally suited to provide accurate estimates of physical observables weighted by the forward flux $\varphi$ at the detectors, which is the solution of the forward Boltzmann linear transport equation [2]. The solution $\varphi^{\dagger}$ of the adjoint Boltzmann equation takes the name of adjoint neutron flux and physically represents the importance of a neutron with respect to a given detector [3]. In terms of the underlying random walks, the adjoint Boltzmann equation corresponds to an inversion of the time arrow, with neutrons flowing backward from the detector and depositing their scores when (and if) they cross or interact with the source [1].

In several applications emerging in radiation shielding and reactor physics, the adjoint approach might yield faster and more accurate answers than the regular forward Monte Carlo approach [1]. In particular, forward particle transport becomes inefficient when the detector is 'small' ${ }^{1}$ in the phase space: in the limit case of a point-like detector, almost no particles can contribute to the simulation, the probability that a forward random walk coming from the source will cross the detector phase space being negligibly small. On the contrary, all adjoint random walks will start from the detector, and there will be a higher chance that they will eventually cross the source (unless the source is also very small). The so-called reciprocity theorem ensures that arbitrary observables can be estimated by resorting to either forward or backward Monte Carlo methods, each leading to the same unbiased score, upon inverting the roles of source and detector [3]. The choice of either scheme must be guided by a variance minimization principle, which is problem-dependent. Ideally, zero-variance Monte Carlo schemes might be conceived by weighting the forward random walks by the importance obtained by solving the corresponding adjoint problem $[1,4,5]$.

The idea of extending standard (i.e., forward) Monte Carlo methods for neutral particle transport to the simulation of backward random walks was first proposed in the pioneering works appeared at the end of the 1960s [6-8], based on an original idea by Maynard [9]. Although initially tailored to solve ad-hoc problems with a limited number of allowed

\footnotetext{
a Corresponding author: andrea.zoia@cea.fr

1 We use the terms 'small' or 'large' referring to the concept of probability to detect a signal.
} 
nuclear reactions, these schemes have been soon generalized to cover arbitrary collision events, scattering distributions and multiplication [10-15]. In particular, it was shown that most of the proposed adjoint Monte Carlo methods can be regarded as particular cases of a broad class of probabilistic models where the simulation of artificial particles (called adjunctons), with modified forward displacement and collision kernels, provides an unbiased estimate of the adjoint flux [15]. Following these theoretical advances, there have been several attempts at implementing adjoint Monte Carlo methods in continuous-energy transport codes [16-19]. Although the key concepts are firmly established [13,14] and can be a priori applied to production Monte Carlo codes [17-19], adjoint particle transport demands that adjuncton displacements and collisions be sampled from the modified kernels: this daunting task requires either extensively modifying existing sampling routines for particle transport [10,15], or rewriting the entire nuclear data pre-processing tools that are currently used for Monte Carlo codes $[14,18,19]$.

Despite these difficulties, adjoint Monte Carlo methods are still a subject of active research per se [20-22] and in view of providing efficient variance reduction strategies for forward methods [23-25]. Bearing in mind the possibility of introducing adjoint methods into TRIPOLI-4 ${ }^{\circledR}$, the production Monte Carlo transport code developed at CEA [26], in this work we illustrate a comparison of different adjoint simulation models. Monte Carlo simulation findings will be tested against benchmark solutions for continuous-energy neutron transport in infinite media, with elastic and inelastic scattering, absorption and multiplication, and their performances will be assessed in terms of both variance and figure of merit.

This paper is organized as follows: in Sec. 2 we will briefly recall the forward and adjoint formulations of linear particle transport, which will set the ground for the derivation of the adjuncton transport models. In Sec. 3 we will describe the benchmark configurations and the reference solutions that we will use in order to compare the various adjoint Monte Carlo methods. Simulation findings will be analysed in Sec. 4 for different collision kernels. The effects of population control techniques on the obtained results will be also discussed. Conclusions will be finally drawn in Sec. 5. Technical details will be condensed in the Appendix.

\section{A class of stochastic models for adjoint neutron transport}

\subsection{The reciprocity property for linear particle transport}

The forward integro-differential form of the Boltzmann equation for steady-state linear particle transport, with a unit point source of neutrons at spatial coordinate $\mathbf{r}_{0}$, monocromatic at energy $E_{0}$ and collimated along direction $\boldsymbol{\Omega}_{0}$, reads as follows:

$$
\begin{array}{r}
\boldsymbol{\Omega} \cdot \nabla G\left(\mathbf{r}_{0}, \boldsymbol{\Omega}_{0}, E_{0} \rightarrow \mathbf{r}, \boldsymbol{\Omega}, E\right)+\Sigma_{t}(\mathbf{r}, E) G\left(\mathbf{r}_{0}, \boldsymbol{\Omega}_{0}, E_{0} \rightarrow \mathbf{r}, \boldsymbol{\Omega}, E\right)= \\
\sum_{j} \iint \nu_{j}\left(E^{\prime}\right) \Sigma_{j}\left(\mathbf{r}, E^{\prime}\right) f_{j}\left(\boldsymbol{\Omega}^{\prime}, E^{\prime} \rightarrow \boldsymbol{\Omega}, E\right) G\left(\mathbf{r}_{0}, \boldsymbol{\Omega}_{0}, E_{0} \rightarrow \mathbf{r}, \boldsymbol{\Omega}^{\prime}, E^{\prime}\right) d \boldsymbol{\Omega}^{\prime} d E^{\prime}+Q_{0},
\end{array}
$$

with $Q_{0}=\delta\left(\mathbf{r}-\mathbf{r}_{0}\right) \delta\left(\Omega-\Omega_{0}\right) \delta\left(E-E_{0}\right)$. The solution $G$ takes the name of Green's function. Here $\Sigma_{t}(\mathbf{r}, E)$ is the total cross section for neutrons at position $\mathbf{r}$ and incident energy $E, \Sigma_{j}(\mathbf{r}, E)$ is the cross section for reaction of type $j$ (such that $p_{j}(E)=\Sigma_{j}(E) / \Sigma_{t}(E)$ is the probability for a neutron with energy $E$ to undergo a reaction event of type $j$ upon collision), $\nu_{j}(E)$ is the mean number of neutrons released in a collision of type $j$, and $f_{j}\left(\boldsymbol{\Omega}^{\prime}, E^{\prime} \rightarrow \boldsymbol{\Omega}, E\right)$ is the (normalized) probability density of transferring the neutron energy from $E^{\prime}$ to $E$ and its direction from $\Omega^{\prime}$ to $\Omega$ in a collision of type $j$. In order to make notation simpler, we will assume that a single isotope is present in the media considered: the generalization to the case of material compositions containing several isotopes is straightforward.

Based on the linear superposition theorem, the angular neutron flux $\varphi_{S}(\mathbf{r}, E, \boldsymbol{\Omega})$ for neutrons at position $\mathbf{r}$, with energy $E$ and direction $\boldsymbol{\Omega}$ for a generic source $S$ over a domain $\mathcal{S}$ of the phase space can be expressed in terms of the forward Green's function as:

$$
\varphi_{S}(\mathbf{r}, \boldsymbol{\Omega}, E)=\iiint_{\mathcal{S}} G\left(\mathbf{r}^{\prime}, \boldsymbol{\Omega}^{\prime}, E^{\prime} \rightarrow \mathbf{r}, \boldsymbol{\Omega}, E\right) S\left(\mathbf{r}^{\prime}, \boldsymbol{\Omega}^{\prime}, E^{\prime}\right) d \mathbf{r}^{\prime} d \boldsymbol{\Omega}^{\prime} d E^{\prime}
$$

where the index $S$ is used to recall that the forward angular neutron flux depends on the source. A generic physical observable $R$ associated to a (real or virtual) detector $D$ is usually formulated as an integral functional of the neutron flux $\varphi_{S}$, namely,

$$
R_{D, S}=\iiint_{\mathcal{D}} \varphi_{S}(\mathbf{r}, \boldsymbol{\Omega}, E) g_{D}(\mathbf{r}, \boldsymbol{\Omega}, E) d \mathbf{r} d \boldsymbol{\Omega} d E,
$$

where $g_{D}$ is the pay-off function of the detector $D$, defined over some domain $\mathcal{D}$ of the phase space. 
The equation adjoint to Eq. (1) reads: ${ }^{2}$

$$
\begin{array}{r}
-\boldsymbol{\Omega} \cdot \nabla G^{\dagger}\left(\mathbf{r}_{0}, \boldsymbol{\Omega}_{0}, E_{0} \rightarrow \mathbf{r}, \boldsymbol{\Omega}, E\right)+\Sigma_{t}(\mathbf{r}, E) G^{\dagger}\left(\mathbf{r}_{0}, \boldsymbol{\Omega}_{0}, E_{0} \rightarrow \mathbf{r}, \boldsymbol{\Omega}, E\right)= \\
\sum_{j} \iint \nu_{j}(E) \Sigma_{j}(\mathbf{r}, E) f_{j}\left(\boldsymbol{\Omega}, E \rightarrow \boldsymbol{\Omega}^{\prime}, E^{\prime}\right) G^{\dagger}\left(\mathbf{r}_{0}, \boldsymbol{\Omega}_{0}, E_{0} \rightarrow \mathbf{r}, \boldsymbol{\Omega}^{\prime}, E^{\prime}\right) d \boldsymbol{\Omega}^{\prime} d E^{\prime}+Q_{0}
\end{array}
$$

whose solution yields the adjoint Green's function $G^{\dagger}[3]$. The forward and adjoint Green's functions in Eqs. (1) and (5) are related by the so-called reciprocity property [3], namely,

$$
G\left(\mathbf{r}^{\prime}, \Omega^{\prime}, E^{\prime} \rightarrow \mathbf{r}, \Omega, E\right)=G^{\dagger}\left(\mathbf{r}, \Omega, E \rightarrow \mathbf{r}^{\prime}, \Omega^{\prime}, E^{\prime}\right)
$$

Using again the linear superposition property, the adjoint angular flux $\varphi_{S^{\dagger}}^{\dagger}$ corresponding to an adjoint source $S^{\dagger}$ over a domain $\mathcal{S}^{\dagger}$ of the phase space can be determined as

$$
\varphi_{S^{\dagger}}^{\dagger}\left(\mathbf{r}_{0}, \boldsymbol{\Omega}_{0}, E_{0}\right)=\iiint_{\mathcal{S}^{\dagger}} G^{\dagger}\left(\mathbf{r}_{0}, \boldsymbol{\Omega}_{0}, E_{0} \rightarrow \mathbf{r}^{\prime}, \boldsymbol{\Omega}^{\prime}, E^{\prime}\right) S^{\dagger}\left(\mathbf{r}^{\prime}, \boldsymbol{\Omega}^{\prime}, E^{\prime}\right) d \mathbf{r}^{\prime} d \boldsymbol{\Omega}^{\prime} d E^{\prime}
$$

where the index $S^{\dagger}$ is used to recall that $\varphi_{S^{\dagger}}^{\dagger}$ depends on the adjoint source. A generic physical observable $R^{\dagger}$ at a (real or virtual) adjoint detector $D^{\dagger}$ over a domain $\mathcal{D}^{\dagger}$ in the phase space can be expressed by taking

$$
R_{D^{\dagger}, S^{\dagger}}^{\dagger}=\iiint_{\mathcal{D}^{\dagger}} \varphi_{S^{\dagger}}^{\dagger}\left(\mathbf{r}_{0}, \boldsymbol{\Omega}_{0}, E_{0}\right) g_{D^{\dagger}}\left(\mathbf{r}_{0}, \boldsymbol{\Omega}_{0}, E_{0}\right) d \mathbf{r}_{0} d \boldsymbol{\Omega}_{0} d E_{0}
$$

where $g_{D^{\dagger}}$ is the pay-off function associated to the adjoint detector.

If we formally identify the adjoint source with the forward detector pay-off function, i.e., $S^{\dagger}=g_{D}$, and the adjoint detector pay-off function with the forward source, i.e., $g_{D^{\dagger}}=S$, we have then

$$
R_{S, D}^{\dagger}=\iiint_{S} \varphi_{D}^{\dagger}\left(\mathbf{r}_{0}, \boldsymbol{\Omega}_{0}, E_{0}\right) S\left(\mathbf{r}_{0}, \boldsymbol{\Omega}_{0}, E_{0}\right) d \mathbf{r}_{0} d \boldsymbol{\Omega}_{0} d E_{0}=\iiint_{D} \varphi_{S}(\mathbf{r}, \boldsymbol{\Omega}, E) g_{D}(\mathbf{r}, \boldsymbol{\Omega}, E) d \mathbf{r} d \boldsymbol{\Omega} d E=R_{D, S}
$$

In other words, the physical observable $R$ in Eq. (3) can be equivalently expressed by resorting to the adjoint flux, based on the reciprocity of the forward and adjoint Green's functions, provided that we set $S^{\dagger}=g_{D}$ and $g_{D^{\dagger}}=S$.

If one is interested in estimating $R$ by Monte Carlo methods, the forward formulation of the linear transport equation given in Eq. (1) suggests that we simulate a forward random walk from the neutron source $S$, with a pay-off function $g_{D}$ for particles entering the generic detector $D$ in the phase space. This is the standard approach that is customarily implemented in production Monte Carlo codes for applications in radiation shielding. The statistical uncertainty of the Monte Carlo estimates decreases with the square root of the number of simulated random walks; if the observable $R$ is distributed over small intervals of one or more variables, the forward Monte Carlo method becomes more and more inefficient, because fewer and fewer random walks cross the detector, and might even become impractical if a quantity at a point detector is sought. On the other hand, Eq. (9) suggests that the quantity $R$ can be alternatively estimated by an adjoint Monte Carlo simulation with backward random walks, the function $g_{D}$ acting as a 'source' and the function $S$ acting as a pay-off function for particles entering the 'detector'. Thus, problems where the estimation function is non-zero in a small volume of the phase space but the neutron source is distributed over a relatively larger volume of the phase space might in principle be efficiently solved by adjoint Monte Carlo methods. However, in the adjoint formulation source and detector are interchanged, and the transport kernels are defined with reversed directions of flight and reverse collision kinematics. This calls for an entirely different approach to the simulation of backward neutron histories.

\subsection{Solving the adjoint transport equation by Monte Carlo methods}

Several approaches have been proposed in order to solve the adjoint neutron transport equation by the Monte Carlo methods. It has been shown that most, if not all, of these schemes might actually be seen as particular cases of a

\footnotetext{
${ }^{2}$ For a well-defined operator associated to a kernel $K\left(z^{\prime} \rightarrow z\right)$, the adjoint kernel $K^{\dagger}$ is defined by the scalar product

$$
\int u(z) \int K\left(z^{\prime} \rightarrow z\right) v\left(z^{\prime}\right) d z^{\prime} d z=\int v(z) \int K^{\dagger}\left(z^{\prime} \rightarrow z\right) u\left(z^{\prime}\right) d z^{\prime} d z
$$

for every set of integrable functions $u(z)$ and $v(z)$. By interchanging the integration variables in Eq. (4), we have thus the definition of the adjoint kernel in terms of the forward kernel, namely, $K^{\dagger}\left(z^{\prime} \rightarrow z\right)=K\left(z \rightarrow z^{\prime}\right)$.
} 
general solution strategy based on the idea of simulating artificial neutron-like particles, called adjunctons, whose forward Green's function can be easily mapped into the adjoint Green's function of the neutrons [15]. In order to set the ground for the following discussion, here we briefly recall the derivation of the transport equation for the adjunctons, by formally transforming the physical quantities associated to the transport of neutrons in the forward formulation.

The main ingredients to simulate a forward random walk are the total macroscopic cross section $\Sigma_{t}(\mathbf{r}, E)$, the macroscopic cross section $\Sigma_{j}(\mathbf{r}, E)$ for a specified reaction $j$, the transfer function $f_{j}\left(\boldsymbol{\Omega}, E \rightarrow \boldsymbol{\Omega}^{\prime}, E^{\prime}\right)$, and the multiplicity $\nu_{j}(E)$ for each reaction $j$. The corresponding cross sections for the adjunctons are formally introduced as:

$$
\tilde{\Sigma}_{j}(\mathbf{r}, E)=\Sigma_{t}(\mathbf{r}, E) \frac{\tilde{p}_{j}(\mathbf{r}, E)}{\sum_{k} \tilde{p}_{k}(\mathbf{r}, E)}
$$

where the quantities $\tilde{p}_{j} \geq 0$ are arbitrary bounded functions and the sum runs over all neutron reaction types [12,15]. The specification of the functions $\tilde{p}_{j}$ implicitly defines an adjoint transport model that can be solved by Monte Carlo simulation, as detailed in the following. The total cross section for the adjunctons stems from Eq. (10), namely,

$$
\tilde{\Sigma}_{t}(\mathbf{r}, E)=\sum_{j} \tilde{\Sigma}_{j}(\mathbf{r}, E)=\Sigma_{t}(\mathbf{r}, E)
$$

and is thus equal to the forward total cross section. The transfer function $\tilde{f}_{j}\left(\boldsymbol{\Omega}^{\prime}, E^{\prime} \rightarrow \boldsymbol{\Omega}, E\right)$ and the multiplicity $\tilde{\nu}_{j}\left(E^{\prime}\right)$ for adjunctons are then defined by the balance relationship:

$$
\tilde{\nu}_{j}\left(E^{\prime}\right) \tilde{\Sigma}_{j}\left(\mathbf{r}, E^{\prime}\right) \tilde{f}_{j}\left(\boldsymbol{\Omega}^{\prime}, E^{\prime} \rightarrow \boldsymbol{\Omega}, E\right)=\nu_{j}(E) \Sigma_{j}(\mathbf{r}, E) f_{j}\left(\boldsymbol{\Omega}, E \rightarrow \boldsymbol{\Omega}^{\prime}, E^{\prime}\right) .
$$

The transfer function $\tilde{f}_{j}$ for adjunctons must be normalized. Equation (12) yields thus the multiplicity

$$
\tilde{\nu}_{j}\left(E^{\prime}\right)=\iint \nu_{j}(E) f_{j}\left(\boldsymbol{\Omega}, E \rightarrow \boldsymbol{\Omega}^{\prime}, E^{\prime}\right) \frac{\Sigma_{j}(\mathbf{r}, E)}{\tilde{\Sigma}_{j}\left(\mathbf{r}, E^{\prime}\right)} d \boldsymbol{\Omega} d E .
$$

Finally, from Eq. (12) we can express the transfer function $\tilde{f}_{j}$ as:

$$
\tilde{f}_{j}\left(\boldsymbol{\Omega}^{\prime}, E^{\prime} \rightarrow \boldsymbol{\Omega}, E\right)=\frac{\nu_{j}(E) f_{j}\left(\boldsymbol{\Omega}, E \rightarrow \boldsymbol{\Omega}^{\prime}, E^{\prime}\right) \Sigma_{j}(\mathbf{r}, E)}{\tilde{\nu}_{j}\left(E^{\prime}\right) \tilde{\Sigma}_{j}\left(\mathbf{r}, E^{\prime}\right)}=\frac{\nu_{j}(E) f_{j}\left(\boldsymbol{\Omega}, E \rightarrow \boldsymbol{\Omega}^{\prime}, E^{\prime}\right) \Sigma_{j}(\mathbf{r}, E)}{\iint \nu_{j}(E) f_{j}\left(\boldsymbol{\Omega}, E \rightarrow \boldsymbol{\Omega}^{\prime}, E^{\prime}\right) \Sigma_{j}(\mathbf{r}, E) d \boldsymbol{\Omega} d E} .
$$

By construction, the transport process for adjunctons is the same as for regular particles in forward linear transport, i.e., free flights and collisions with scattering, multiplication and/or absorption [12, 15]. Hence, adjunctons formally satisfy a Boltzmann-like forward transport equation. In particular, their Green's function $\tilde{G}$ obeys

$$
\begin{array}{r}
\boldsymbol{\Omega} \cdot \nabla \tilde{G}\left(\mathbf{r}_{0}, \boldsymbol{\Omega}_{0}, E_{0} \rightarrow \mathbf{r}, \boldsymbol{\Omega}, E\right)+\tilde{\Sigma}_{t}(\mathbf{r}, E) \tilde{G}\left(\mathbf{r}_{0}, \boldsymbol{\Omega}_{0}, E_{0} \rightarrow \mathbf{r}, \boldsymbol{\Omega}, E\right)= \\
\sum_{j} \iint \tilde{\nu}_{j}\left(E^{\prime}\right) \tilde{\Sigma}_{j}\left(\mathbf{r}, E^{\prime}\right) \tilde{f}_{j}\left(\boldsymbol{\Omega}^{\prime}, E^{\prime} \rightarrow \boldsymbol{\Omega}, E\right) \tilde{G}\left(\mathbf{r}_{0}, \boldsymbol{\Omega}_{0}, E_{0} \rightarrow \mathbf{r}, \boldsymbol{\Omega}^{\prime}, E^{\prime}\right) d \boldsymbol{\Omega}^{\prime} d E^{\prime}+Q_{0},
\end{array}
$$

which yields the formal relation

$$
G^{\dagger}\left(\mathbf{r}_{0}, \boldsymbol{\Omega}_{0}, E_{0} \rightarrow \mathbf{r}, \boldsymbol{\Omega}, E\right)=\tilde{G}\left(\mathbf{r}_{0},-\boldsymbol{\Omega}_{0}, E_{0} \rightarrow \mathbf{r},-\boldsymbol{\Omega}, E\right)
$$

provided that the boundary conditions for the two Green's functions are consistent [15]. In other words, solving the forward equation for the Green's function of adjunctons leads to the ajoint Green's function of neutrons, as from the transformation in Eq. (16).

Equation (10) formally defines a broad class of forward stochastic models that can be in principle solved by Monte Carlo methods. In order to explicitly simulate adjunctons, one must however be able to sample particle displacement and collision events from the kernels introduced above, which in turn depend on the choice of the functions $\tilde{p}_{j}$. Instead of performing analog Monte Carlo sampling from $\tilde{\Sigma}_{j}, \tilde{f}_{j}$ and $\tilde{\nu}_{j}$, it is also possible to introduce statistical weights for the adjunctons, similarly to what is customarily done for neutrons in forward transport. The adjuncton weights after each collision would be modified to

$$
w^{\prime}=w \frac{\tilde{\nu}_{j}\left(E^{\prime}\right) \tilde{f}_{j}\left(\boldsymbol{\Omega}^{\prime}, E^{\prime} \rightarrow \boldsymbol{\Omega}, E\right)}{\tilde{h}_{j}\left(\boldsymbol{\Omega}^{\prime}, E^{\prime} \rightarrow \boldsymbol{\Omega}, E\right)}=w \frac{\nu_{j}(E) \Sigma_{j}(\mathbf{r}, E) f_{j}\left(\boldsymbol{\Omega}, E \rightarrow \boldsymbol{\Omega}^{\prime}, E^{\prime}\right)}{\tilde{\Sigma}_{j}\left(\mathbf{r}, E^{\prime}\right) \tilde{h}_{j}\left(\boldsymbol{\Omega}^{\prime}, E^{\prime} \rightarrow \boldsymbol{\Omega}, E\right)},
$$


where $w$ is the statistical weight of an adjuncton entering a collision and the function $\tilde{h}_{j}$ is an arbitrary distribution normalized over the same domain as $\tilde{f}_{j}$ [15]. Following the collision event of type $j$, we can thus either sample $\tilde{\nu}_{j}\left(E^{\prime}\right)$ adjunctons from the law $\tilde{f}_{j}$, each with a statistical weight $w=1$, or a single adjuncton with a weight $w^{\prime}$ from the law $\tilde{h}_{j}$. The role of $\tilde{h}_{j}$ is such that an importance sampling can be performed by drawing the adjuncton features from this distribution instead of the original kernel $\tilde{f}_{j}$, which may simplify the calculations and reduce the variance. Following the introduction of statistical weights, population control techniques such as Russian roulette or splitting can be introduced as well: comments will be provided in Sec. 4.5.

In view of comparing the performances of adjoint Monte Carlo schemes, we recall here a few relevant choices for the model functions $\tilde{p}_{j}$ that have been introduced in the literature. In selecting those schemes, we have been mainly guided by their flexibility and broad applicability to arbitrary reaction types, having in mind the possibility of implementing them in the production Monte Carlo code TRIPOLI- $4{ }^{\circledR}$.

The first two schemes $\left(\mathcal{M}_{1}\right.$ and $\left.\mathcal{M}_{2}\right)$ have been proposed by Hoogenboom [13], and differ only by the appearance of an $E^{\prime} / E$ biasing factor in energy. In this case, the two model functions are defined as

$$
\tilde{p}_{j}^{\mathcal{M}_{1}}\left(\mathbf{r}, E^{\prime}\right)=\iint \nu_{j}(E) \Sigma_{j}(\mathbf{r}, E) f_{j}\left(-\boldsymbol{\Omega}, E \rightarrow-\boldsymbol{\Omega}^{\prime}, \rightarrow E^{\prime}\right) d \boldsymbol{\Omega} d E
$$

and

$$
\tilde{p}_{j}^{\mathcal{M}_{2}}\left(\mathbf{r}, E^{\prime}\right)=\iint \nu_{j}(E) \Sigma_{j}(\mathbf{r}, E) \frac{E^{\prime}}{E} f_{j}\left(-\boldsymbol{\Omega}, E \rightarrow-\boldsymbol{\Omega}^{\prime}, \rightarrow E^{\prime}\right) d \boldsymbol{\Omega} d E .
$$

The additional term 1/ $E$ appearing in Eq. (19) can be understood as a biasing factor based on the consideration that the shape of the forward flux in media where elastic scattering is the dominant reaction is of the kind $\varphi(E) \sim 1 / E$ : model $\mathcal{M}_{2}$ can be then regarded as an improvement of $\mathcal{M}_{1}$ with biased adjuncton cross sections having an approximation of the forward flux as a weighting function. The functional forms for $\mathcal{M}_{1}$ and $\mathcal{M}_{2}$ are general and apply to any type of reaction $j$.

In a recent paper [22], a model $\left(\mathcal{M}_{3}\right)$ in which the functions $\tilde{p}_{j}$ are equal to the forward macroscopic cross section of the $j$-th reaction has been proposed, which yields

$$
\tilde{p}_{j}^{\mathcal{M}_{3}}\left(\mathbf{r}, E^{\prime}\right)=\Sigma_{j}\left(\mathbf{r}, E^{\prime}\right) .
$$

This definition applies to any arbitrary reaction $j$ as in the previous models.

The last model $\left(\mathcal{M}_{4}\right)$ that we will consider in this work has been introduced by De Matteis and Simonini [15]. Contrary to the previous schemes, here the definition of the functions $\tilde{p}_{j}$ depends on the considered reaction, although it is in principle possible to cover the entire range of allowed events by carefully deriving ad hoc choices. For instance, for the elastic scattering reaction we would have

$$
\tilde{p}_{e}^{\mathcal{M}_{4}}\left(\mathbf{r}, E^{\prime}\right)=\Sigma_{e}\left(\mathbf{r}, E^{\prime}\right),
$$

as in the previous model, whereas for other reactions different rules would be assigned.

In the following, we will provide an extensive comparison of the adjoint Monte Carlo methods presented above on benchmark problems, and analyse the performances of the corresponding simulation results.

\section{Energy-dependent transport in infinite media}

In order to establish benchmark solutions for the adjoint Monte Carlo schemes, a convenient choice is to resort to neutron transport in infinite media, where $\varphi$ and $\varphi^{\dagger}$ only depend on energy. In this case, the steady-state linear adjoint particle transport equation for the adjoint flux $\varphi^{\dagger}$ reads:

$$
\Sigma_{t}(E) \varphi^{\dagger}(E)=\sum_{j} \nu_{j}(E) \Sigma_{j}(E) \int_{0}^{\infty} f_{j}\left(E \rightarrow E^{\prime}\right) \varphi^{\dagger}\left(E^{\prime}\right) d E^{\prime}+S^{\dagger}(E) .
$$

For the sake of simplicity, let us assume that the particles are generated at a given energy $E_{0}$, i.e.,

$$
S^{\dagger}(E)=S_{0}^{\dagger} \delta\left(E-E_{0}\right),
$$

$S_{0}^{\dagger}$ being a normalization constant. Correspondingly, the adjoint flux can be decomposed into a singular term (due to un-collided contributions), plus a continuous portion (due to collided contributions), namely,

$$
\varphi^{\dagger}(E)=\varphi_{0}^{\dagger} \delta\left(E-E_{0}\right)+\varphi_{c}^{\dagger}(E) .
$$


The energy domain ranges from the source energy of the particles $E_{0}$ up to a cut-off energy, say $E^{*}$. An analytical solution to Eq. (22) has been explicitly derived for the case of elastic scattering alone with general cross-section $\Sigma_{e}(E)[22]$, based on adjoint Placzek functions [27]. The forward transfer function for elastic scattering reads

$$
f_{e}\left(E \rightarrow E^{\prime}\right)=\frac{1}{E(1-\alpha)} \quad \text { for } \quad \alpha E \leq E^{\prime} \leq E
$$

where

$$
\alpha=\left(\frac{A-1}{A+1}\right)^{2}
$$

and $A$ is the ratio of the atomic mass of the nuclide to the neutron mass [2]. Let us suppose that the cross sections do not depend on energy, and that the allowed reactions are elastic scattering and absorption. Then Eq. (22) yields:

$$
\varphi^{\dagger}(E)=\frac{\gamma}{E(1-\alpha)} \int_{\alpha E}^{E} \varphi^{\dagger}(E) d E^{\prime}+\frac{S^{\dagger}(E)}{\Sigma_{t}},
$$

with $\gamma=\Sigma_{e} / \Sigma_{t} \leq 1$. By equating singular parts, from Eq. (27) we immediately have

$$
\varphi_{0}^{\dagger}=\frac{S_{0}^{\dagger}}{\Sigma_{t}}
$$

The collided portion of the adjoint flux satisfies the integral equation

$$
\varphi_{c}^{\dagger}(E)=\frac{\theta\left(E-E_{0}\right) \theta\left(E_{0}-\alpha E\right)}{E(1-\alpha)} \frac{S_{0}^{\dagger}}{\Sigma_{t}}+\frac{\gamma}{E(1-\alpha)} \int_{\alpha E}^{E} \varphi_{c}^{\dagger}\left(E^{\prime}\right) d E^{\prime},
$$

where $\theta$ is the Heaviside function. The solution of Eq. (29) can be recursively obtained by closely following the arguments in [22], and yields:

$$
\begin{aligned}
\varphi_{c, 1}^{\dagger}(E) & =\frac{\gamma}{E_{0} \Sigma_{t}(1-\alpha)}\left(\frac{E}{E_{0}}\right)^{\frac{\gamma}{1-\alpha}-1} \\
\varphi_{c, 2}^{\dagger}(E) & =\frac{\gamma^{2}}{E_{0} \Sigma_{t}(1-\alpha)^{2}}\left(\frac{E}{E_{0}}\right)^{\frac{\gamma}{1-\alpha}-1}\left[\left(\frac{1-\alpha}{\gamma}\right)\left(1-\alpha^{\frac{\gamma}{1-\alpha}}\right)-\alpha^{\frac{\gamma}{1-\alpha}} \log \frac{\alpha E}{E_{0}}\right] \\
& \vdots \\
\varphi_{c, n}^{\dagger}(E) & =\frac{\gamma^{n}}{E_{0} \Sigma_{t}(1-\alpha)^{n}}\left(\frac{E}{E_{0}}\right)^{\frac{\gamma}{1-\alpha}-1}\left[\left(\frac{1-\alpha}{\gamma}\right)^{n-1}\left(1-\alpha^{\frac{\gamma}{1-\alpha}}\right)-\left(\frac{1-\alpha}{\gamma}\right)^{n-2} \alpha^{\frac{\gamma}{1-\alpha}} \log \frac{\alpha E}{E_{0}}+\right. \\
& \left.+\sum_{m=2}^{n-1}(-1)^{m-2} \alpha^{\frac{\gamma m}{1-\alpha}}\left(\frac{((1-\alpha) / \gamma)^{n-m}}{(m-1) !} \log ^{m-1} \frac{\alpha^{m} E}{E_{0}}+\frac{((1-\alpha) / \gamma)^{n-m-1}}{m !} \log ^{m} \frac{\alpha^{m} E}{E_{0}}\right)\right]
\end{aligned}
$$

where the $n$-th term $\varphi_{c, n}^{\dagger}(E)$ is non-zero only in the energy range

$$
\frac{E_{0}}{\alpha^{n-1}} \leq E \leq \frac{E_{0}}{\alpha^{n}}
$$

The adjoint flux and its derivatives show discontinuities similar to those appearing in the forward solution (the so-called Placzek transients [27]) and $\varphi_{c}^{\dagger}(E)$ asymptotically converges to a constant value for large $E \gg E_{0}$.

When other kinds of reactions are involved, with arbitrary transition kernels, analytical expressions are no longer available, but it is nonetheless relatively straightforward to derive reference solutions for Eq. (22) by accurate numerical integration. We start from a guess function $\varphi_{0}^{\dagger}$ and at each iteration $q$ we compute

$$
\varphi_{q+1}^{\dagger}(E)=\sum_{j} \nu_{j}(E) \frac{\Sigma_{j}(E)}{\Sigma_{t}(E)} \int_{0}^{\infty} f_{j}\left(E \rightarrow E^{\prime}\right) \varphi_{q}^{\dagger}\left(E^{\prime}\right) d E^{\prime}+\frac{S^{\dagger}(E)}{\Sigma_{t}(E)}
$$

This procedure is repeated until a given convergence criterion is satisfied. 


\section{Monte Carlo simulation results}

The adjoint flux $\varphi^{\dagger}(E)$, solution of Eq. (22) for a point-source located at $E_{0}$, is now determined by Monte Carlo methods. The four adjuncton models introduced above have been implemented in a computer code and tested against reference solutions obtained as detailed in Sec. 3. The energy domain is divided into 100 equally spaced energy intervals between $E_{0}$ and the cut-off energy $E^{*}$, and each interval acts as a detector for $\varphi^{\dagger}(E)$. The adjuncton histories are simulated as follows.

1. The first step consists in sampling the initial energy from the adjuncton source. For models $\mathcal{M}_{1}, \mathcal{M}_{3}$ and $\mathcal{M}_{4}$, the initial energy $E$ is sampled from the normalized distribution

$$
\frac{S^{\dagger}(E)}{\int S^{\dagger}\left(E^{\prime}\right) d E^{\prime}}
$$

whereas for model $\mathcal{M}_{2}$ the initial energy $E$ is sampled from

$$
\frac{S^{\dagger}(E)}{E \int \frac{S^{\dagger}\left(E^{\prime}\right)}{E^{\prime}} d E^{\prime}}
$$

Correspondingly, for models $\mathcal{M}_{1}, \mathcal{M}_{3}$ and $\mathcal{M}_{4}$, set the score weight $w_{\text {score }}=\int S^{\dagger}\left(E^{\prime}\right) d E^{\prime}$, which for the pointsource yields $w_{\text {score }}=1$. For model $\mathcal{M}_{2}$, set the score weight $w_{\text {score }}=\int S^{\dagger}\left(E^{\prime}\right) / E^{\prime} d E^{\prime}$, which for the point-source yields $w_{\text {score }}=1 / E_{0}$. For all models, set the initial statistical weight of the adjunctons $w=1$.

2. Sample a free flight from an exponential distribution with average $\Sigma_{t}^{-1}(E)$.

3. Score the adjoint neutron flux by using a collision estimator, for each event occurring in a detector interval. For models $\mathcal{M}_{1}, \mathcal{M}_{3}$ and $\mathcal{M}_{4}$, this corresponds to recording

$$
w_{\text {score }} \frac{w}{\Sigma_{t}(E)},
$$

whereas for model $\mathcal{M}_{2}$ we have to record

$$
w_{\text {score }} E \frac{w}{\Sigma_{t}(E)} .
$$

4. Sample a reaction $j$ with probability $\tilde{\Sigma}_{j} / \tilde{\Sigma}_{t}$. If required, apply population control techniques. If the particle has not been killed (because of absorption or population control), sample the energy of the emitted particle from distribution $\tilde{f}_{j}$ (or $\tilde{h}_{j}$ if variance reduction techniques are applied). Update then the statistical weight of the adjuncton by using Eq. (17).

This procedure is iterated until the adjuncton has disappeared or the history has been terminated because the current particle energy is above the simulation cut-off. A total number of $N$ adjuncton histories are simulated. Finally, the computed scores are divided by the total initial weight of the simulated adjunctons, and by the energy interval $\Delta E$ of each energy bin.

The relative variances of the Monte Carlo scores will be estimated from

$$
\sigma_{r}^{2}=\sum_{b}\left(\frac{\sigma_{b}}{\mu_{b}}\right)^{2}
$$

where the sum runs over all energy intervals, $\mu_{b}$ is the average score in interval $b$ and $\sigma_{b}$ is the corresponding standard deviation. The figure of merit is thus estimated as

$$
F O M=\frac{1}{\sigma_{r}^{2} T_{c}},
$$

where $T_{c}$ is the computational time. Four different neutron reactions are considered for our tests: elastic scattering, inelastic scattering, absorption and $(n, 2 n)$ multiplication. In the following, we detail the simulation procedures that apply to each collision event according to the specific Monte Carlo models. 


\subsection{Elastic scattering}

Our first benchmark problem concerns adjoint neutron transport inside a purely elastic scattering medium, with constant cross section $\Sigma_{e}$ (and multiplicity $\nu_{e}=1$ ) and a point source at $E_{0}$. We now explicitly derive the rules for the stochastic models introduced above. For model $\mathcal{M}_{1}$, as shown in $[13,14]$, from Eq. (18) we have

$$
\tilde{p}_{e}^{\mathcal{M}_{1}}=\Sigma_{e} \frac{\log \alpha}{\alpha-1}
$$

whence also $\tilde{\Sigma}_{e}=\Sigma_{e}$ and

$$
\tilde{\nu}_{e}=\frac{\log \alpha}{\alpha-1}
$$

from Eq. (13). Finally, the energy after collision is selected from

$$
\tilde{f}_{e}\left(E^{\prime} \rightarrow E\right)=-\frac{1}{E \log \alpha} \quad \text { for } \quad E^{\prime} \leq E \leq \frac{E^{\prime}}{\alpha},
$$

which follows from Eq. (14). For the statistical weights it is assumed that $\tilde{h}_{e}\left(E^{\prime} \rightarrow E\right)=\tilde{f}_{e}\left(E^{\prime} \rightarrow E\right)$, i.e., no biasing is applied to the scattering law, so that from Eq. (17) we have

$$
w^{\prime}=w \frac{\log \alpha}{\alpha-1} .
$$

Since $\alpha<1$, the statistical weights of adjunctons are bound to increase at each elastic scattering collision [13,14].

For model $\mathcal{M}_{2}$, as shown in [13,14], from Eq. (19) we have

$$
\tilde{p}_{e}^{\mathcal{M}_{2}}=\Sigma_{e}
$$

with $\tilde{\Sigma}_{e}=\Sigma_{e}$. The multiplication factor yields

$$
\tilde{\nu}_{e}=\frac{\log \alpha}{\alpha-1}
$$

from Eq. (13). The biased scattering law is taken to be [13]

$$
\tilde{h}_{e}\left(E^{\prime} \rightarrow E\right)=\frac{E^{\prime}}{E^{2}(1-\alpha)} \quad \text { for } \quad E^{\prime} \leq E \leq \frac{E^{\prime}}{\alpha} .
$$

Correspondingly, from Eq. (17) we have the statistical weight

$$
w^{\prime}=w \frac{E}{E^{\prime}} .
$$

In this case, the statistical weights of adjunctons are multiplied by a factor $E / E^{\prime}$ at each elastic scattering collision, independently of $\alpha$.

For elastic scattering, model $\mathcal{M}_{3}$ is defined such that $\tilde{p}_{e}^{\mathcal{M}_{3}}=\Sigma_{e}$ and $\tilde{\Sigma}_{e}=\Sigma_{e}[22]$. The scattering law and the weight multiplication are also equivalent to those of model $\mathcal{M}_{1}$.

Finally, for model $\mathcal{M}_{4}$ we have $\tilde{p}_{e}^{\mathcal{M}_{4}}=\Sigma_{e}$, with $\tilde{\Sigma}_{e}=\Sigma_{e}$ [15]. The definition of model $\mathcal{M}_{4}$ is formally equal to model $\mathcal{M}_{2}$. However, in model $\mathcal{M}_{4}$ the source particles are emitted from the natural adjoint distribution and have a weight multiplier at score equal to $w_{\text {score }}=1$ [15], whereas in model $\mathcal{M}_{2}$ the particles are sampled from a biased source and have a weight multiplier at score equal to $w_{\text {score }}=1 / E_{0}[13]$.

For each model we have simulated $10^{8}$ adjuncton histories. We have assumed $\Sigma_{e}=1$ and $E_{0}=1$. The maximum allowed energy of the simulated particles is taken to be $E^{*}=E_{0} / \alpha^{4}$, depending on the medium. Reference solutions for $\varphi^{\dagger}(E)$ have been derived over an energy grid with $10^{4}$ points from Eq. (30). For illustration, an example of Monte Carlo estimation for the adjoint flux is shown in Fig. 1, where we compare the simulation result corresponding to model $\mathcal{M}_{1}$ to the reference solution, for $A=5$. A good agreement is observed, and similar unbiased results have been obtained also for models $\mathcal{M}_{2}$ and $\mathcal{M}_{4}$. In order to compare the performances of the methods tested here, we have run several simulations for different values of $A$ : the relative standard deviations and the figures of merit (FOM) integrated over the entire energy range are displayed in Tabs. 1 and 2, respectively. As $A$ increases, the FOM for models $\mathcal{M}_{2}$ and $\mathcal{M}_{4}$ converge to similar values, larger than those of model $\mathcal{M}_{1}$. This can be understood by observing that for model $\mathcal{M}_{1}$ elastic scattering collisions lead to a relevant change in the particle weight, by a factor $\log \alpha /(\alpha-1)$ depending on $A$. For model $\mathcal{M}_{4}$, the weight change does not explicitly depend on $\alpha$ and is given by the ratio $E / E^{\prime}$. Finally, for model $\mathcal{M}_{4}$, the particles do not change their weights. For small $A$, model $\mathcal{M}_{2}$ displays the largest FOM due to lower statistical weights and the biased scattering law $\tilde{h}_{e}$. Model $\mathcal{M}_{4}$ performs better than $\mathcal{M}_{1}$, thanks to the use of the biased scattering law. Finally, it is worth observing that model $\mathcal{M}_{2}$ shows the largest variance for each value of $A$ examined here: the best FOM is achieved thanks to the shortest simulation times. 


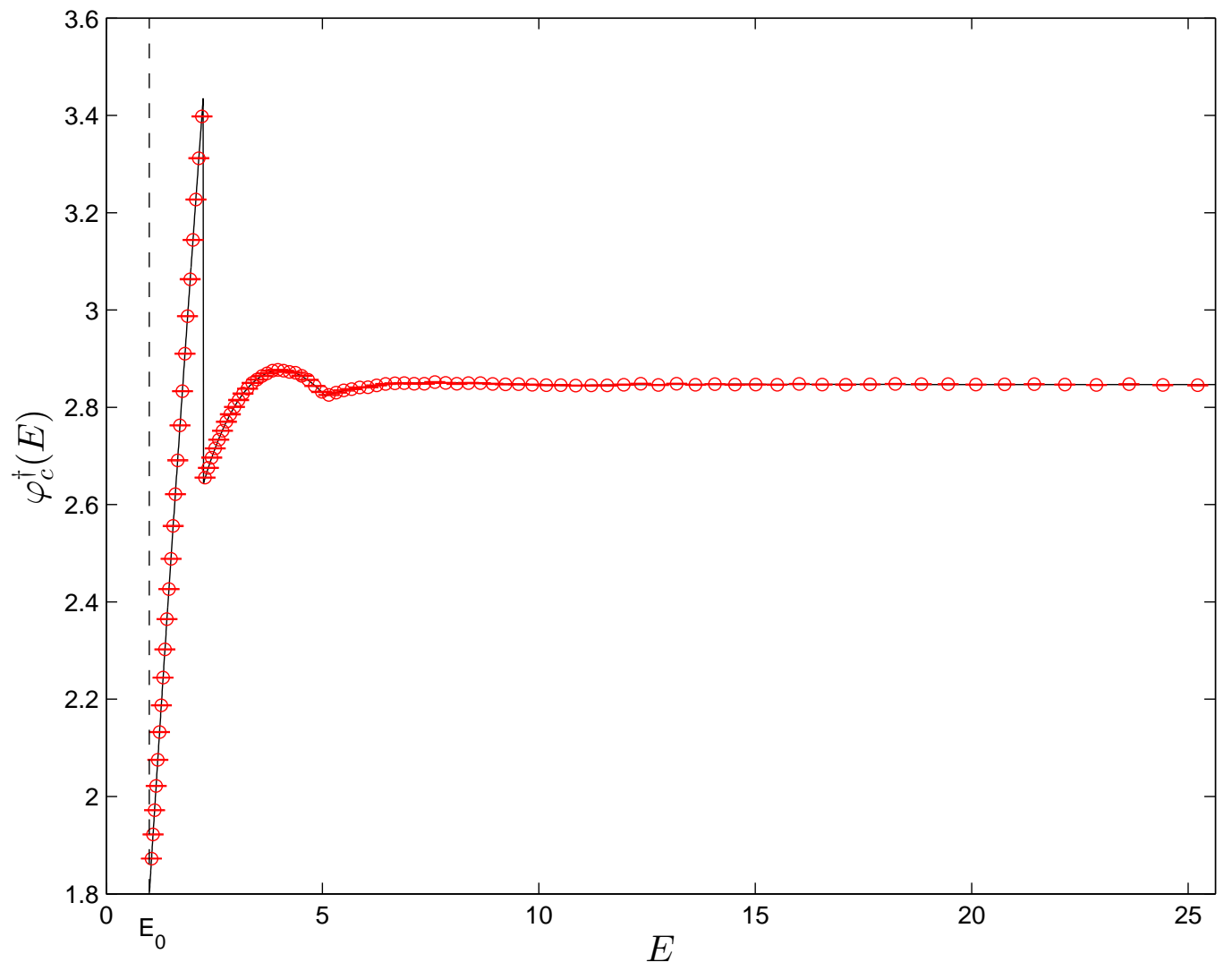


Table 1. Relative variances $\sigma_{r}^{2}$ of the Monte Carlo scores, from Eq. (37), for a purely elastic scattering medium.

\begin{tabular}{cccc}
\hline$A$ & $\mathcal{M}_{1,3}$ & $\mathcal{M}_{2}$ & $\mathcal{M}_{4}$ \\
\hline 5 & $6.2 \times 10^{-6}$ & $1.1 \times 10^{-5}$ & $5.6 \times 10^{-6}$ \\
15 & $1.1 \times 10^{-5}$ & $1.2 \times 10^{-5}$ & $1.1 \times 10^{-5}$ \\
25 & $1.3 \times 10^{-5}$ & $1.2 \times 10^{-5}$ & $1.2 \times 10^{-5}$ \\
\hline
\end{tabular}

Table 2. FOM of the Monte Carlo scores, from Eq. (38), for a purely elastic scattering medium.

\begin{tabular}{cccc}
\hline$A$ & $\mathcal{M}_{1,3}$ & $\mathcal{M}_{2}$ & $\mathcal{M}_{4}$ \\
\hline 5 & $8.0 \times 10^{1}$ & $4.3 \times 10^{2}$ & $1.2 \times 10^{2}$ \\
15 & $2.3 \times 10^{2}$ & $4.3 \times 10^{2}$ & $3.6 \times 10^{2}$ \\
25 & $2.7 \times 10^{2}$ & $4.1 \times 10^{2}$ & $4.4 \times 10^{2}$ \\
\hline
\end{tabular}
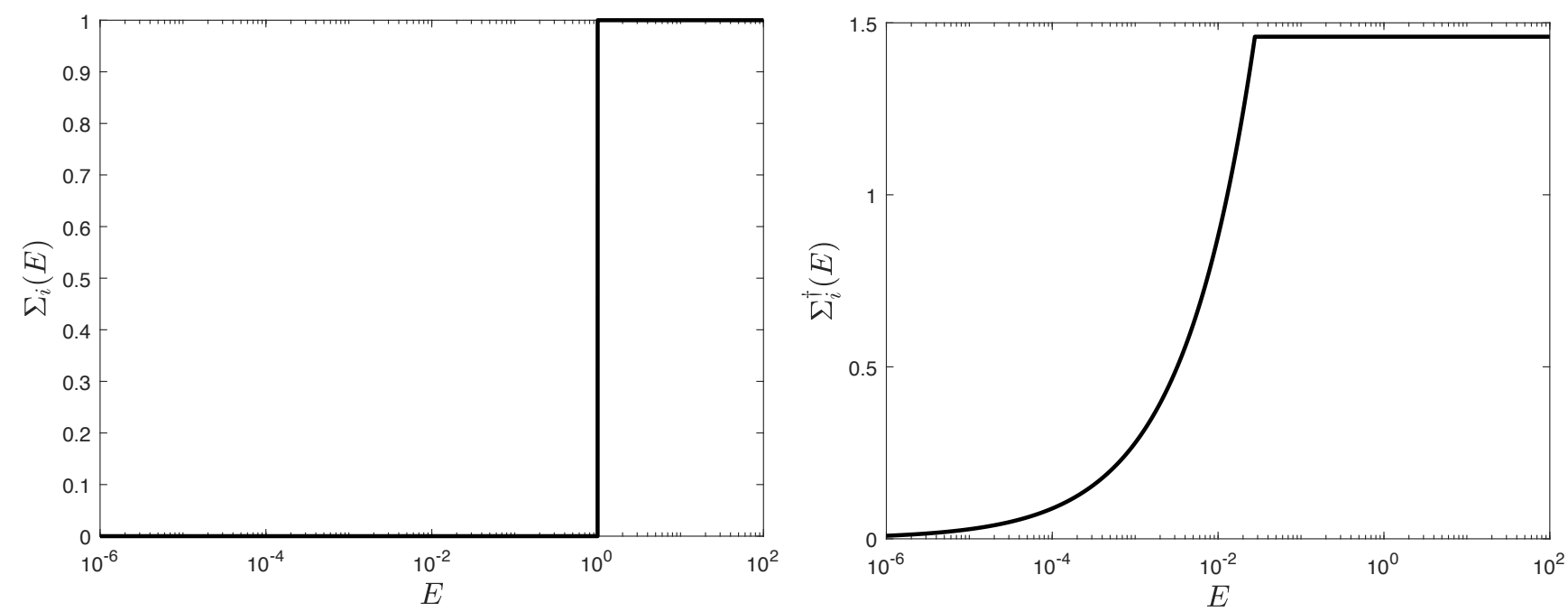

Fig. 2. Left: macroscopic inelastic cross section $\Sigma_{i}$, with threshold at $\epsilon_{i}=1$ and post-threshold value $\Sigma_{i}=1$. Right: adjuncton cross section $\tilde{\Sigma}_{i}$ for model $\mathcal{M}_{1}$, corresponding to the same physical parameters as for the case on the left. Here we have taken $A=5$.

\subsection{Inelastic scattering}

As a second benchmark configuration we have considered a system with elastic and inelastic scattering, with constant cross sections $\Sigma_{e}$ and $\Sigma_{i}$, respectively. The law for inelastic scattering reads

$$
f_{i}\left(E \rightarrow E^{\prime}\right)=\frac{1}{E(1-\alpha) \sqrt{1-\epsilon_{i} / E}}
$$


where $\epsilon_{i}$ is the threshold energy for the reaction [26]. The post-reaction energy $E^{\prime}$ lies in the interval

$$
\left(\frac{A \sqrt{1-\epsilon_{i} / E}-1}{A+1}\right)^{2} E \leq E^{\prime} \leq\left(\frac{A \sqrt{1-\epsilon_{i} / E}+1}{A+1}\right)^{2} E .
$$

The associated multiplicity is trivially $\nu_{i}=1$. As shown in [13], the functions $\tilde{p}_{i}$ for models $\mathcal{M}_{1}$ and $\mathcal{M}_{2}$ read

$$
\tilde{p}_{i}^{\mathcal{M}_{1}}\left(E^{\prime}\right)=\int_{E_{i, \min }^{\prime}}^{E_{i, \max }^{\prime}} \Sigma_{i} \frac{1}{E(1-\alpha) \sqrt{1-\epsilon_{i} / E}} d E
$$

from Eq. (18), and

$$
\tilde{p}_{i}^{\mathcal{M}_{2}}\left(E^{\prime}\right)=\int_{E_{i, \text { min }}^{\prime}}^{E_{i, \max }^{\prime}} \Sigma_{i} \frac{E^{\prime}}{E^{2}(1-\alpha) \sqrt{1-\epsilon_{i} / E}} d E,
$$

from Eq. (19), respectively. Here $E_{i, \min }^{\prime}$ and $E_{i, \max }^{\prime}$ are the lowest and the highest energies of the scattered particle, whose explicit expressions are derived in the Appendix. Since the inelastic scattering is a threshold reaction, by definition $\Sigma_{i}(E)=0$ for $E<\epsilon_{i}$. However, the adjuncton cross sections $\Sigma_{i}^{\dagger}$ that follow from the functions $\tilde{p}_{i}$ for model $\mathcal{M}_{1}$ and $\mathcal{M}_{2}$ do not vanish. A comparison between forward and direct cross sections for $\mathcal{M}_{1}$ is shown in Fig. 2 . From Eq. (13), the adjuncton multiplicity reads

$$
\tilde{\nu}_{i}\left(E^{\prime}\right)=\int f_{i}\left(E \rightarrow E^{\prime}\right) \frac{\Sigma_{i}(E)}{\Sigma_{t}\left(E^{\prime}\right)}\left[1+\frac{\tilde{p}_{e}\left(E^{\prime}\right)}{\tilde{p}_{i}\left(E^{\prime}\right)}\right] d E .
$$

The ratio $\tilde{p}_{e}\left(E^{\prime}\right) / \tilde{p}_{i}\left(E^{\prime}\right)$ appearing in Eq. (51) is well defined for every value of the energy $E^{\prime}$, which stems from the integration boundaries in the definition of $\tilde{p}_{i}$ for models $\mathcal{M}_{1}$ or $\mathcal{M}_{2}$. For model $\mathcal{M}_{1}$, the scattering law for adjunctons is $\tilde{f}_{i}$, defined as in Eq. (14), whereas for model $\mathcal{M}_{2}$ we sample from the biased law $\tilde{h}_{i}=\left(E^{\prime} / E\right) \tilde{f}_{i}$.

For model $\mathcal{M}_{3}$, the case of inelastic scattering was not considered in [22]. Following the general definition provided above, we should have

$$
\tilde{p}_{i}^{\mathcal{M}_{3}}\left(E^{\prime}\right)=\Sigma_{i}\left(E^{\prime}\right)
$$

which yields

$$
\tilde{\Sigma}_{i}\left(E^{\prime}\right)=\Sigma_{t}\left(E^{\prime}\right) \frac{\tilde{p}_{i}\left(E^{\prime}\right)}{\tilde{p}_{i}\left(E^{\prime}\right)+\tilde{p}_{e}\left(E^{\prime}\right)} .
$$

According to this definition, for model $\mathcal{M}_{3}$ the ratio $\tilde{p}_{e}\left(E^{\prime}\right) / \tilde{p}_{i}\left(E^{\prime}\right)$ appearing in Eq. (51) would be defined only for $E^{\prime} \geq \epsilon_{i}$. In order to allow for threshold reactions in model $\mathcal{M}_{3}$ the multiplicity factor can be, for instance, defined by using the functions $\tilde{p}_{i}$ of model $\mathcal{M}_{1}$ when $E^{\prime}<\epsilon_{i}$, and those of model $\mathcal{M}_{3}$ when $E^{\prime} \geq \epsilon_{i}$. For model $\mathcal{M}_{3}$, the inelastic scattering law for adjunctons is $\tilde{f}_{i}$, defined as in Eq. (14).

Finally, by following [15] in model $\mathcal{M}_{4}$ we have

$$
\tilde{p}_{i}^{\mathcal{M}_{4}}\left(E^{\prime}\right)=\frac{\Sigma_{i}(\bar{E})}{(1-\alpha)} \sqrt{1-\frac{\epsilon_{i}}{\bar{E}}} I\left(E^{\prime}\right) E^{\prime}
$$

where the function $I\left(E^{\prime}\right)$ is defined as

$$
I\left(E^{\prime}\right)=\int_{E_{\mathrm{L}}}^{E_{\mathrm{R}}} \frac{d E}{E^{2}\left(1-\epsilon_{s i} / E\right)}=\frac{1}{\epsilon_{i}} \log \left(\frac{E_{L}}{E_{R}} \frac{E_{R}-\epsilon_{i}}{E_{L}-\epsilon_{i}}\right)
$$

and the quantity $\bar{E}$ is provided in the Appendix. The quantities $E_{L}$ and $E_{R}$ denote the lowest and the highest allowed energies, respectively, whose definitions are given in the Appendix. The sampling of the scattered adjuncton is made from a biased law

$$
\tilde{h}_{i}\left(E^{\prime} \rightarrow E\right)=\frac{1}{I\left(E^{\prime}\right)} \frac{1}{E^{2}\left(1-\epsilon_{i} / E\right)} \quad \text { for } E_{L} \leq E \leq E_{R}
$$

For our numerical comparisons, a point source is set at $E_{0}=1$ and the maximum allowed energy of the simulated particles is taken to be $E^{*}=E_{0} / \alpha^{4}$. The viable energy range is partitioned into 100 equally spaced intervals, and the adjoint flux determined by Monte Carlo methods is benchmarked against reference solutions computed from Eq. (32) over a grid with $10^{4}$ points with 30 iterations. The threshold energy for inelastic scattering has been set to $\epsilon_{i}=1.5$, and we have taken $A=25$. A parametric study has been performed by changing the ratio $\Sigma_{i} / \Sigma_{t}$ of the inelastic scattering cross section with respect to the total cross section in the region $E>\epsilon_{i}$ : three pairs of values have been 


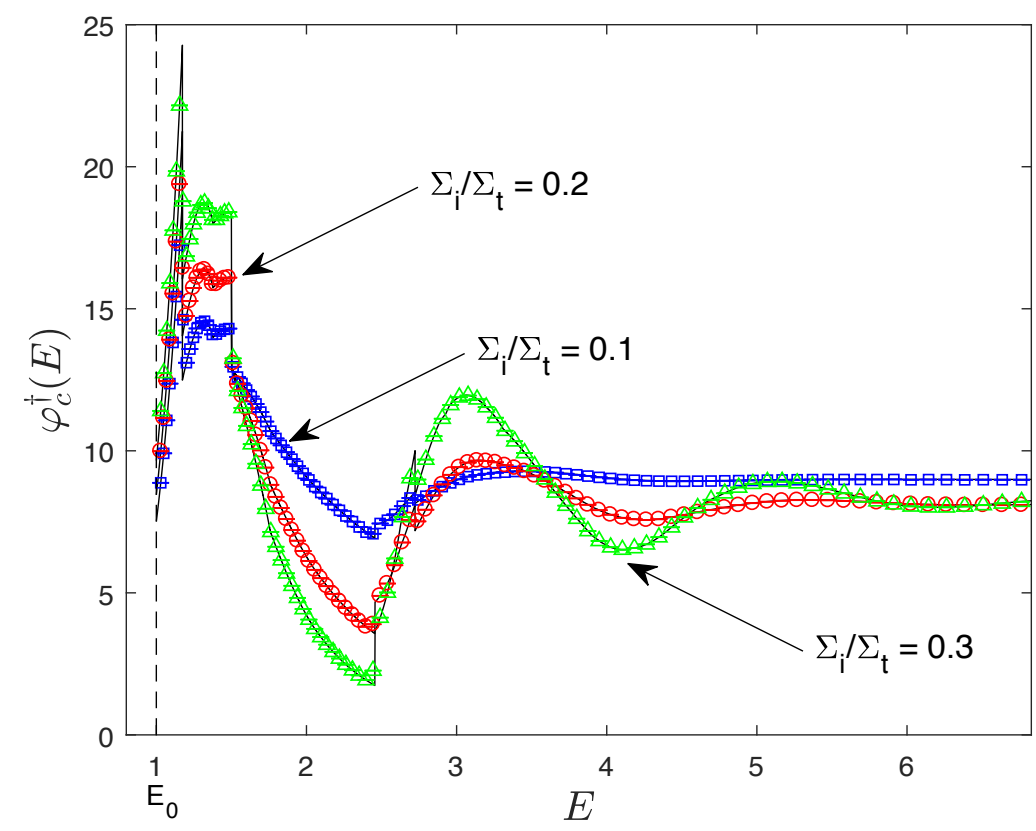

Fig. 3. Adjoint collided flux $\varphi_{c}^{\dagger}(E)$ for a medium with elastic and inelastic scattering, with $A=25$. The Monte Carlo results corresponding to model $\mathcal{M}_{1}$ are displayed with symbols. Blue squares: $\Sigma_{i}=0.1$ and $\Sigma_{e}=0.9$; red circles: $\Sigma_{i}=0.2$ and $\Sigma_{e}=0.8$; green triangles: $\Sigma_{i}=0.3$ and $\Sigma_{e}=0.7$. The reference solution from Eq. (32) is displayed with a solid line.

Table 3. Relative variances $\sigma_{r}^{2}$ of the Monte Carlo scores, from Eq. (37), for a medium with elastic and inelastic scattering.

\begin{tabular}{ccccc}
\hline$\Sigma_{i} / \Sigma_{t}$ & $\mathcal{M}_{1}$ & $\mathcal{M}_{2}$ & $\mathcal{M}_{3}$ & $\mathcal{M}_{4}$ \\
\hline 0.1 & $3.7 \times 10^{-6}$ & $5.6 \times 10^{-6}$ & $3.7 \times 10^{-6}$ & $3.2 \times 10^{-6}$ \\
0.2 & $5.0 \times 10^{-6}$ & $7.6 \times 10^{-6}$ & $5.0 \times 10^{-6}$ & $4.2 \times 10^{-6}$ \\
0.3 & $6.9 \times 10^{-6}$ & $8.8 \times 10^{-6}$ & $6.9 \times 10^{-6}$ & $5.1 \times 10^{-6}$ \\
\hline
\end{tabular}

considered, namely, $\Sigma_{i}=0.1, \Sigma_{e}=0.9 ; \Sigma_{i}=0.2, \Sigma_{e}=0.8 ; \Sigma_{i}=0.3, \Sigma_{e}=0.7$. In each case, $\Sigma_{t}=1$ for $E>\epsilon_{i}$. For illustration, an example of calculation is displayed in Fig. 3 for model $\mathcal{M}_{1}$. A good agreement is observed, and similar unbiased results have been obtained also for models $\mathcal{M}_{2}, \mathcal{M}_{3}$ and $\mathcal{M}_{4}$. The qualitative behaviour is similar to that of elastic scattering alone, but the amplitude of the Placzek transients increases with increasing $\Sigma_{i} / \Sigma_{t}$.

The comparison of the different Monte Carlo models for various values of $\Sigma_{i} / \Sigma_{t}$ is displayed in Tab. 3 for the relative standard deviation and in Tab. 4 for the FOM, integrated over the entire energy range. The behaviour of these configurations is similar to those with pure elastic scattering. Concerning variance, the increase of the incidence of inelastic scattering leads to increasing statistical weights: model $\mathcal{M}_{2}$ shows the largest value for variance, followed by $\mathcal{M}_{4}$, and finally $\mathcal{M}_{1}$ and $\mathcal{M}_{3}$. However, similarly as for the case of pure elastic scattering, model $\mathcal{M}_{2}$ leads to shorter simulation times, and thus yields the largest FOM. Model $\mathcal{M}_{4}$, whose kernel has been tailored specifically for inelastic reactions with threshold, shows also very good performances. Methods $\mathcal{M}_{1}$ and $\mathcal{M}_{3}$ have the lowest FOM for this set of problems. 
Table 4. FOM of the Monte Carlo scores, from Eq. (38), for a medium with elastic and inelastic scattering.

\begin{tabular}{ccccc}
\hline$\Sigma_{i} / \Sigma_{t}$ & $\mathcal{M}_{1}$ & $\mathcal{M}_{2}$ & $\mathcal{M}_{3}$ & $\mathcal{M}_{4}$ \\
\hline 0.1 & $9.1 \times 10^{1}$ & $2.7 \times 10^{2}$ & $8.7 \times 10^{1}$ & $1.6 \times 10^{2}$ \\
0.2 & $7.3 \times 10^{1}$ & $2.2 \times 10^{2}$ & $6.7 \times 10^{1}$ & $1.4 \times 10^{2}$ \\
0.3 & $4.9 \times 10^{1}$ & $1.6 \times 10^{2}$ & $4.6 \times 10^{1}$ & $1.0 \times 10^{2}$ \\
\hline
\end{tabular}

Table 5. Relative variances $\sigma_{r}^{2}$ of the Monte Carlo scores, from Eq. (37), for a medium with elastic scattering and absorption.

\begin{tabular}{ccccc}
\hline$\Sigma_{i} / \Sigma_{t}$ & $\mathcal{M}_{1}$ & $\mathcal{M}_{2}$ & $\mathcal{M}_{3}$ & $\mathcal{M}_{4}$ \\
\hline 0.1 & $8.1 \times 10^{-6}$ & $1.7 \times 10^{-5}$ & $9.0 \times 10^{-6}$ & $7.1 \times 10^{-6}$ \\
0.3 & $1.3 \times 10^{-5}$ & $8.6 \times 10^{-5}$ & $2.5 \times 10^{-5}$ & $1.5 \times 10^{-5}$ \\
0.5 & $5.9 \times 10^{-5}$ & $7.0 \times 10^{-4}$ & $1.2 \times 10^{-4}$ & $5.8 \times 10^{-5}$ \\
\hline
\end{tabular}

\subsection{Absorption}

We now consider the contribution of absorption to the transport of adjunctons. As discussed in [13,14], for model $\mathcal{M}_{1}$ we have

$$
\tilde{p}_{a}^{\mathcal{M}_{1}}\left(E^{\prime}\right)=0
$$

because no particles are emitted from an absorption $\left(\nu_{a}=0\right)$. This definition leads to $\tilde{\Sigma}_{a}=0$, which means that absorption cannot be sampled explicitly. The effects of absorptions are nonetheless conveyed by the other reactions via the total cross section and the multiplicity factor. For instance, for a medium with elastic scattering (with cross section $\Sigma_{e}$ ) and absorption, from Eq. (13) the multiplicity of elastic scattering would read

$$
\tilde{\nu}_{e}=\frac{\Sigma_{e}}{\Sigma_{t}} \frac{\log \alpha}{\alpha-1}
$$

i.e., lower by a factor $\Sigma_{e} / \Sigma_{t}$ than in the case of pure elastic scattering. Thus, the adjuncton statistical weight would be reduced by the same amount at each collision, namely,

$$
w^{\prime}=w \frac{\Sigma_{e}}{\Sigma_{t}} \frac{\log \alpha}{\alpha-1}
$$

which stems from Eq. (17) with $\tilde{f}_{e}=\tilde{h}_{e}$. The same considerations apply to method $\mathcal{M}_{2}$.

Concerning model $\mathcal{M}_{3}$, no multiplicity factor is present in the general expression for $\tilde{p}_{a}$ [22], i.e.,

$$
\tilde{p}_{a}^{\mathcal{M}_{3}}=\Sigma_{a}
$$

Absorption can then be explicitly sampled for adjunctons, with $\tilde{\Sigma}_{a}=\Sigma_{a}$. If the adjuncton is absorbed, the particle history is terminated. Model $\mathcal{M}_{4}$ coincides with the prescriptions of model $\mathcal{M}_{3}[15]$.

For our numerical comparisons, the same energy range and discretization are assumed as in the previous case, and the adjoint flux determined by Monte Carlo methods is benchmarked against reference solutions computed from Eq. (30) over a grid with $10^{4}$ points. We have taken a medium with elastic scattering and absorption, with $A=5$. A parametric study has been performed by changing the ratio $\Sigma_{a} / \Sigma_{t}$ of the absorption cross section with respect to the total cross section: three pairs of values have been considered, namely, $\Sigma_{a}=0.1, \Sigma_{e}=0.9 ; \Sigma_{a}=0.3, \Sigma_{e}=0.7$; $\Sigma_{a}=0.5, \Sigma_{e}=0.5$. In each case $\Sigma_{t}=1$. For illustration, an example of calculation is displayed in Fig. 4 for model $\mathcal{M}_{1}$. A good agreement is observed, and similar unbiased results have been obtained also for models $\mathcal{M}_{2}, \mathcal{M}_{3}$ and $\mathcal{M}_{4}$. When the fraction of absorption is small, the qualitative behaviour is similar to that of elastic scattering alone; however, due to absorption, adjunctons disappear and the adjoint flux vanishes in the limit of large energies $E \gg E_{0}$. As expected, the adjoint flux decreases faster for increasing values of the ratio $\Sigma_{a} / \Sigma_{t}$.

The comparison of the different Monte Carlo models for various values of $\Sigma_{a} / \Sigma_{t}$ is displayed in Tab. 5 for the relative standard deviation and in Tab. 6 for the FOM, integrated over the entire energy range. The behaviour of these configurations is different from those with pure elastic scattering, and the discrepancies increase with an increasing fraction of absorption. Model $\mathcal{M}_{1}$ shows the largest FOM, followed by $\mathcal{M}_{4}, \mathcal{M}_{3}$, and finally, $\mathcal{M}_{2}$. Since the shape of the forward flux for a medium with a large absorption cross section deviates from the $1 / E$ behaviour, the performance gain induced by the biasing factor of model $\mathcal{M}_{2}$ deteriorates. This feature is also mirrored by the variance, as reported in Tab. 5 , where $\mathcal{M}_{2}$ displays the largest variance, followed by $\mathcal{M}_{3}, \mathcal{M}_{4}$, and $\mathcal{M}_{1}$. 


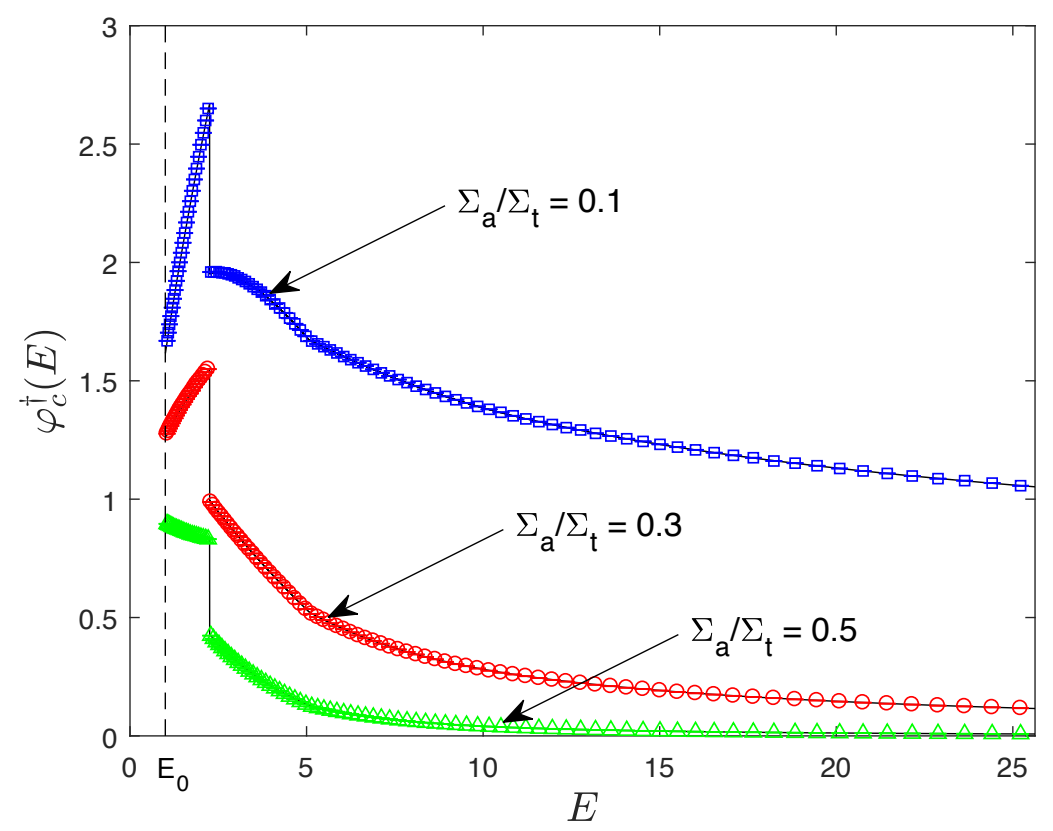

Fig. 4. Adjoint collided flux $\varphi_{c}^{\dagger}(E)$ for a medium with elastic scattering and absorption, with $A=5$. The Monte Carlo results corresponding to model $\mathcal{M}_{1}$ are displayed with symbols. Blue squares: $\Sigma_{a}=0.1$ and $\Sigma_{e}=0.9 ;$ red circles: $\Sigma_{a}=0.3$ and $\Sigma_{e}=0.7$; green triangles: $\Sigma_{a}=0.5$ and $\Sigma_{e}=0.5$. The reference solution from Eq. (30) is displayed with a solid line.

Table 6. FOM of the Monte Carlo scores, from Eq. (38), for a medium with elastic scattering and absorption.

\begin{tabular}{ccccc}
\hline$\Sigma_{i} / \Sigma_{t}$ & $\mathcal{M}_{1}$ & $\mathcal{M}_{2}$ & $\mathcal{M}_{3}$ & $\mathcal{M}_{4}$ \\
\hline 0.1 & $1.4 \times 10^{2}$ & $2.8 \times 10^{2}$ & $1.2 \times 10^{2}$ & $2.2 \times 10^{2}$ \\
0.3 & $2.8 \times 10^{2}$ & $6.0 \times 10^{1}$ & $1.6 \times 10^{2}$ & $3.1 \times 10^{2}$ \\
0.5 & $1.3 \times 10^{2}$ & $7.3 \times 10^{0}$ & $8.8 \times 10^{1}$ & $8.1 \times 10^{1}$ \\
\hline
\end{tabular}

\subsection{Multiplication}

To conclude our analysis, we examine the case of a multiplication reaction of the kind $(n, 2 n)$, i.e., with multiplicity $\nu_{m}=2$. Generalization to the case of $(n, x n)$ reactions or more broadly to fission would be relatively straightforward. For the sake of simplicity, we assume that particles are re-emitted with an evaporation spectrum as

$$
f_{m}\left(E \rightarrow E^{\prime}\right) \propto E^{\prime} \exp \left(-E^{\prime} / \xi(E)\right) \quad \text { for } \quad 0<E^{\prime}<E-\epsilon_{m}
$$

where $\epsilon_{m}$ is the threshold energy for the reaction, and $\xi(E)$ is the so-called nuclear temperature, which depends on the energy of the neutron before the collision [28]. A common modelling choice is that $\xi(E)$ follows a linear relation with respect to energy, namely, $\xi(E)=E-\epsilon_{m}$. In this case we have

$$
f_{m}\left(E \rightarrow E^{\prime}\right)=\frac{e}{e-2} \frac{E^{\prime}}{\left(E-\epsilon_{m}\right)^{2}} e^{-\frac{E^{\prime}}{E-\epsilon_{m}}} \text { for } 0<E^{\prime}<E-\epsilon_{m}
$$


Suppose that the $(n, 2 n)$ cross section $\Sigma_{m}$ is constant and that $\epsilon_{m}=0$. Correspondingly, for model $\mathcal{M}_{1}$ from Eq. (18) we have

$$
\tilde{p}_{m}^{\mathcal{M}_{1}}=2 \Sigma_{m} \frac{e-1}{e-2}
$$

where we have assumed that the largest energy of a particle emitted from a $(n, 2 n)$ reaction is unbounded for increasing incident energy $E$. The adjuncton multiplicity factor is then obtained from Eq. (13) as

$$
\tilde{\nu}_{m}\left(E^{\prime}\right)=2 \frac{e-1}{e-2} \frac{\Sigma_{m}}{\tilde{\Sigma}_{m}\left(E^{\prime}\right)}
$$

As a consequence, the adjoint multiplicity $\tilde{\nu}_{m}$ depends on the exiting energy $E^{\prime}$ and can largely deviate from 2 . A finer analysis with finite $E_{\max }$ for the emitted particles and non-vanishing threshold $\epsilon_{m}>0$ would show that $\tilde{\nu}_{m}$ becomes small for $E^{\prime}$ close to $E_{\max }$ and for small values of $\epsilon_{m}$. The energy distribution for the re-emitted adjunctons follows from Eq. (14) as:

$$
\tilde{f}_{m}\left(E^{\prime} \rightarrow E\right)=\frac{e}{e-1} \frac{E^{\prime}}{E^{2}} e^{-\frac{E^{\prime}}{E}} \text { for } E>E^{\prime}
$$

The sampling is made from the unbiased distribution $\tilde{f}_{m}$, so that for the statistical weight from Eq. (17) we have

$$
w^{\prime}=2 w \frac{e-1}{e-2} \frac{\Sigma_{m}}{\tilde{\Sigma}_{m}\left(E^{\prime}\right)} .
$$

For model $\mathcal{M}_{2}$, from Eq. (19) we have

$$
\tilde{p}_{e}^{\mathcal{M}_{2}}=2 \Sigma_{m}
$$

The energy of the re-emitted particle is sampled from the biased distribution

$$
\tilde{h}_{m}=\frac{e}{2(e-2)} \frac{E^{\prime 2}}{E^{3}} e^{-\frac{E^{\prime}}{E}} \quad \text { for } E>E^{\prime} .
$$

Models $\mathcal{M}_{3}$ and $\mathcal{M}_{4}$ follow the rules of $\mathcal{M}_{1}$, but with

$$
\tilde{p}_{m}=\tilde{\Sigma}_{m}
$$

For our numerical comparisons, the same energy range and discretization is assumed as in the previous case, and the adjoint flux determined by Monte Carlo methods is benchmarked against reference solutions computed from Eq. (32) over a grid with $10^{5}$ points and 25 iterations. We consider a medium with $(n, 2 n)$, elastic scattering and absorption, with $A=5$. For the sake of simplicity, we have set $\epsilon_{m}=0$. A parametric study has been performed by changing the weight of the $(n, 2 n)$ cross section with respect to the total cross section: three pairs of values have been considered, namely, $\Sigma_{m}=0.09, \Sigma_{a}=0.01 ; \Sigma_{m}=0.05, \Sigma_{a}=0.05 ; \Sigma_{m}=0.01, \Sigma_{a}=0.09$. In each case, we have taken $\Sigma_{e}=0.9$, so that $\Sigma_{t}=1$. For illustration, an example of calculation is displayed in Fig. 5 for model $\mathcal{M}_{1}$. A good agreement is observed also for this problem, and similar unbiased results have been obtained for models $\mathcal{M}_{2}, \mathcal{M}_{3}$ and $\mathcal{M}_{4}$. For a ratio $\Sigma_{m} /\left(\Sigma_{m}+\Sigma_{a}\right)=0.5$, the flux eventually converges to an asymptotic value for large $E$ (the effects of particle productions and particle losses are compensated). For larger ratios, the flux diverges for large $E$, and conversely for smaller ratios the flux converges to zero.

The comparison of the different Monte Carlo models for various values of $\Sigma_{m} /\left(\Sigma_{m}+\Sigma_{a}\right)$ is displayed in Tab. 7 for the relative standard deviation and in Tab. 8 for the FOM, integrated over the entire energy range. The behaviour for these configurations is very similar to those with pure elastic scattering. This can be justified by recalling that for these configurations $\Sigma_{e} / \Sigma_{t}=0.9$. The FOM for models $\mathcal{M}_{2}$ and $\mathcal{M}_{4}$ is similar, and larger than those of models $\mathcal{M}_{1}$ and $\mathcal{M}_{3}$. Model $\mathcal{M}_{2}$ displays the largest figure of merit due to lower statistical weights and the biased scattering law $\tilde{h}_{e}$. Model $\mathcal{M}_{2}$ shows the largest variance and displays the best FOM thanks to the shortest simulation times. Variances and FOMs tend to increase for larger fractions of the $(n, 2 n)$ reaction.

\subsection{Population control and variance reduction techniques}

In order to reduce the variance of the scores, several population control techniques and variance reduction schemes have been tested in the Monte Carlo simulations illustrated in the previous section. Implicit capture allows neutrons to survive absorption by reducing their statistical weight by a factor of $1-\tilde{\Sigma}_{a} / \tilde{\Sigma}_{t}$. For infinite media, particles cannot escape the simulated medium and would thus live forever in the absence of a cut-off energy. It is thus convenient to use implicit capture in combination with Russian roulette, a fair game that applies to the statistical weights of the 


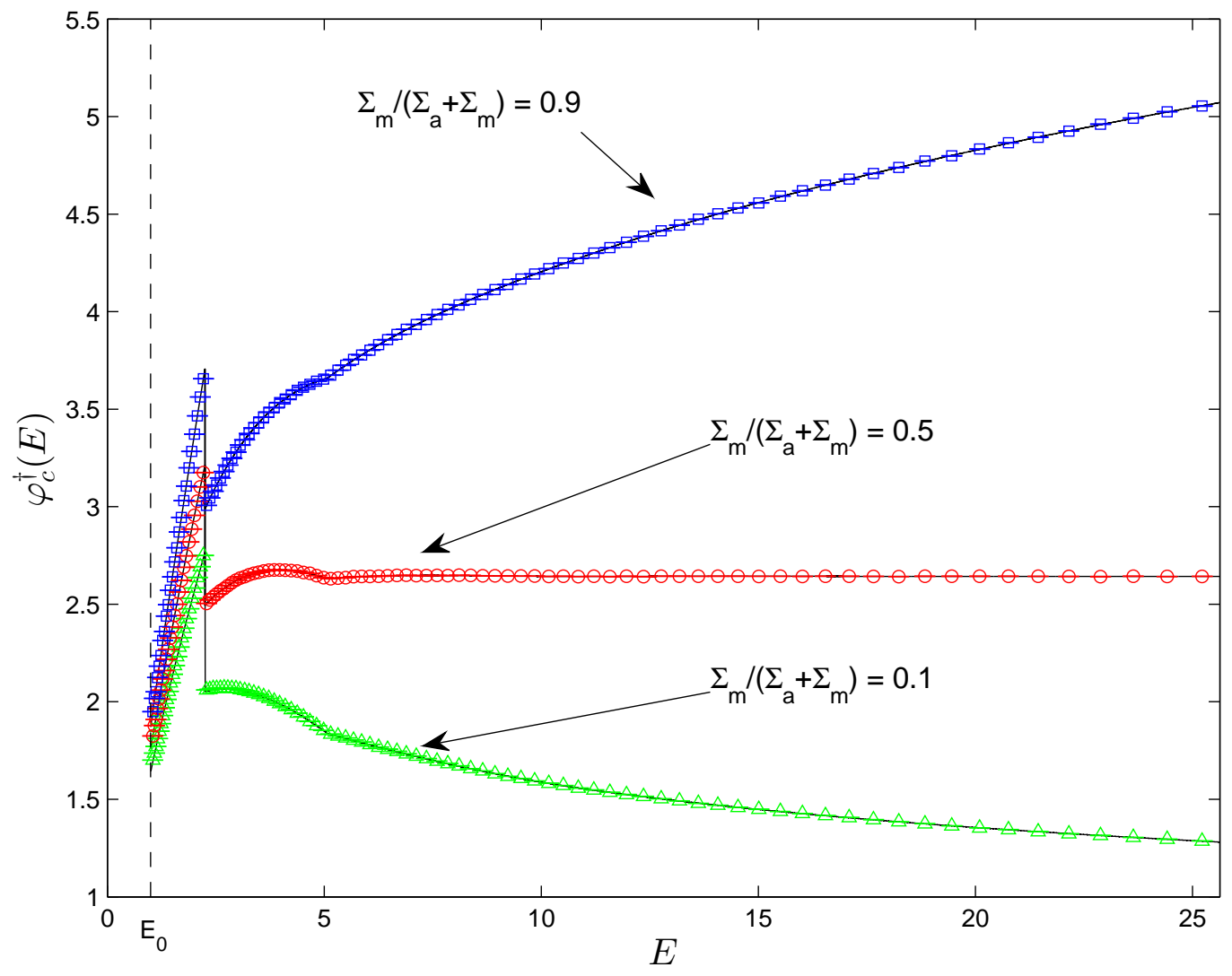


Table 7. Relative variances $\sigma_{r}^{2}$ of the Monte Carlo scores, from Eq. (37), for a medium with elastic scattering, absorption and $(n, 2 n)$ multiplication.

\begin{tabular}{ccccc}
\hline$\Sigma_{m} /\left(\Sigma_{m}+\Sigma_{a}\right)$ & $\mathcal{M}_{1}$ & $\mathcal{M}_{2}$ & $\mathcal{M}_{3}$ & $\mathcal{M}_{4}$ \\
\hline 0.1 & $7.3 \times 10^{-6}$ & $1.2 \times 10^{-5}$ & $7.8 \times 10^{-6}$ & $6.1 \times 10^{-6}$ \\
0.5 & $7.6 \times 10^{-6}$ & $1.2 \times 10^{-5}$ & $8.4 \times 10^{-6}$ & $6.3 \times 10^{-6}$ \\
0.9 & $8.0 \times 10^{-6}$ & $1.6 \times 10^{-5}$ & $8.8 \times 10^{-6}$ & $6.9 \times 10^{-6}$ \\
\hline
\end{tabular}

Table 8. FOM of the Monte Carlo scores, from Eq. (38), for a medium with elastic scattering, absorption and ( $n, 2 n)$ multiplication.

\begin{tabular}{ccccc}
\hline$\Sigma_{m} /\left(\Sigma_{m}+\Sigma_{a}\right)$ & $\mathcal{M}_{1}$ & $\mathcal{M}_{2}$ & $\mathcal{M}_{3}$ & $\mathcal{M}_{4}$ \\
\hline 0.1 & $2.3 \times 10^{1}$ & $1.8 \times 10^{2}$ & $3.6 \times 10^{1}$ & $3.1 \times 10^{1}$ \\
0.5 & $3.8 \times 10^{1}$ & $2.1 \times 10^{2}$ & $5.7 \times 10^{1}$ & $5.1 \times 10^{1}$ \\
0.9 & $6.2 \times 10^{1}$ & $2.0 \times 10^{2}$ & $9.7 \times 10^{1}$ & $8.3 \times 10^{1}$ \\
\hline
\end{tabular}

Table 9. Relative variances $\sigma_{r}^{2}$ of the Monte Carlo scores, from Eq. (37), for a purely elastic scattering medium: the effects of particle splitting.

\begin{tabular}{ccccc}
\hline$A$ & splitting & $\mathcal{M}_{1}$ and $\mathcal{M}_{3}$ & $\mathcal{M}_{2}$ & $\mathcal{M}_{4}$ \\
\hline 5 & yes & $6.2 \times 10^{-6}$ & $1.1 \times 10^{-5}$ & $5.6 \times 10^{-6}$ \\
5 & no & $1.8 \times 10^{-5}$ & $1.1 \times 10^{-5}$ & $1.1 \times 10^{-5}$ \\
15 & yes & $1.1 \times 10^{-5}$ & $1.2 \times 10^{-5}$ & $1.1 \times 10^{-5}$ \\
15 & no & $1.3 \times 10^{-5}$ & $1.2 \times 10^{-5}$ & $1.2 \times 10^{-5}$ \\
25 & yes & $1.3 \times 10^{-5}$ & $1.2 \times 10^{-5}$ & $1.2 \times 10^{-5}$ \\
25 & no & $1.3 \times 10^{-5}$ & $1.2 \times 10^{-5}$ & $1.2 \times 10^{-5}$ \\
\hline
\end{tabular}

Table 10. FOM of the Monte Carlo scores, from Eq. (38), for a purely elastic scattering medium: the effects of particle splitting.

\begin{tabular}{ccccc}
\hline$A$ & splitting & $\mathcal{M}_{1}$ and $\mathcal{M}_{3}$ & $\mathcal{M}_{2}$ & $\mathcal{M}_{4}$ \\
\hline 5 & yes & $8.0 \times 10^{1}$ & $4.3 \times 10^{2}$ & $1.2 \times 10^{2}$ \\
5 & no & $2.1 \times 10^{2}$ & $4.7 \times 10^{2}$ & $4.7 \times 10^{2}$ \\
15 & yes & $2.3 \times 10^{2}$ & $4.3 \times 10^{2}$ & $3.6 \times 10^{2}$ \\
15 & no & $2.95 \times 10^{2}$ & $4.7 \times 10^{2}$ & $5.1 \times 10^{2}$ \\
25 & yes & $2.7 \times 10^{2}$ & $4.1 \times 10^{2}$ & $4.4 \times 10^{2}$ \\
25 & no & $2.9 \times 10^{2}$ & $4.7 \times 10^{2}$ & $5.1 \times 10^{2}$ \\
\hline
\end{tabular}

particles $w^{\prime}$ after collision. A threshold $0<\omega_{R}<1$ is set for the statistical weights. If $w^{\prime}<\omega_{R}$, a random number $\xi$ is uniformly sampled in the interval $[0,1]$. If $\xi<\omega_{R}$, the particle history is terminated. Otherwise, $w=1$ and the history is allowed to continue. Russian roulette is an effective variance reduction technique, in that it removes particles with lower weights, which contribute less to the scores. The threshold has been set to $\omega_{R}=0.8$ for our simulations.

Conversely, the statistical weight of the particles can rapidly increase when the multiplicity factor is larger than one and non-analog Monte Carlo methods are applied. In this case, population control can be enforced by resorting to particle splitting. If the particle weight $w^{\prime}$ after collision is larger than some threshold $\omega_{S}, n_{b}-1$ copies of the original particle are created, with $n_{b}$ defined as the largest integer value that is not larger than the original weight $w^{\prime}$, and a new weight equal to $w^{\prime} / n_{b}$ is assigned to the original particle and to its copies. For our simulations, we have set $\omega_{S}=2$.

Let us consider for instance the case of purely elastic scattering presented in Sec. 4.1. For the different Monte Carlo models presented above, we have $w_{\mathcal{M}_{1}, \mathcal{M}_{3}}^{\prime}=w \log \alpha /(\alpha-1), w_{\mathcal{M}_{2}}^{\prime}=w$, and $w_{\mathcal{M}_{4}}^{\prime}=w\left(E / E^{\prime}\right)$. In Fig. 6 we illustrate the Monte Carlo simulation results for model $\mathcal{M}_{1}$ and $A=5$, with and without particle splitting. For these simulations we have used $10^{6}$ particles. As expected, when splitting is applied the variance of the scores decreases, since for this particular case particles have an average weight multiplier larger than unit, which would lead to a large weight dispersion. We have examined the behaviour of particle splitting as a function of $A$; it is worth recalling that increasing $A$ leads to lower weight multipliers at elastic collisions. Results are presented in Tab. 9 for the relative standard deviation and in Tab. 10 for the FOM, integrated over the entire energy range. Splitting is never applied for model $\mathcal{M}_{2}$, since the statistical weight does not change after the collision. On the other hand, methods $\mathcal{M}_{1}, \mathcal{M}_{3}$ and 


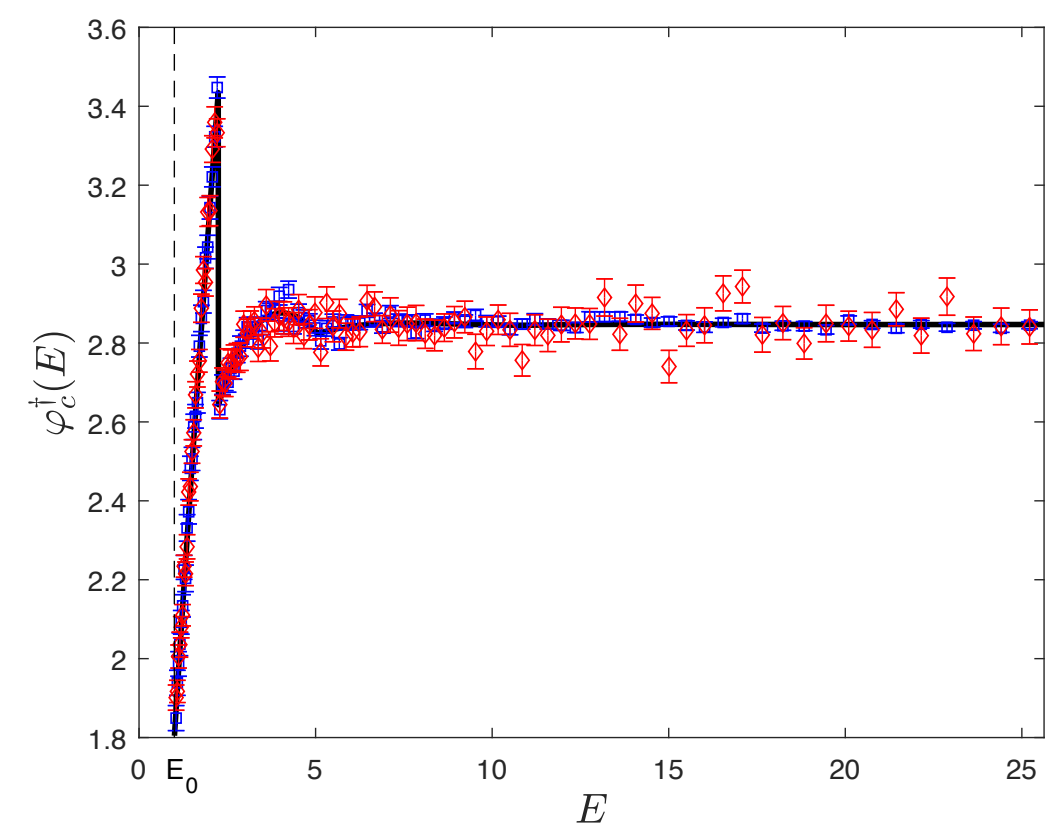

Fig. 6. Adjoint collided flux $\varphi_{c}^{\dagger}(E)$ for a medium with elastic scattering, with $A=5$. The Monte Carlo results corresponding to model $\mathcal{M}_{1}$ are displayed with symbols. Blue squares: simulation with particle splitting. Red diamonds: simulation without particle splitting. The reference solution from Eq. (30) is displayed with a solid line.

$\mathcal{M}_{4}$ imply particle weights increasing at each elastic collision. For models $\mathcal{M}_{1}$ and $\mathcal{M}_{3}$, the weight multiplier depends only on the collided nucleus mass and is always larger than unit; for model $\mathcal{M}_{4}$ the weight multiplier depends on the energy ratio $E / E^{\prime}$ and is thus again larger than one, since adjunctons are scattered to higher energies. In particular, from Eq. (45) it is possible to evaluate the average energy sampled from $\tilde{h}_{e}$ for model $\mathcal{M}_{4}$, namely,

$$
\bar{E}=\int E \tilde{h}_{e}\left(E^{\prime} \rightarrow E\right) d E=E^{\prime} \frac{\log \alpha}{\alpha-1}
$$

For the average weight multiplier of model $\mathcal{M}_{4}$ we thus obtain

$$
\bar{w}_{\mathcal{M}_{4}}^{\prime}=w \frac{\bar{E}}{E^{\prime}}=w \frac{\log \alpha}{\alpha-1}
$$

Therefore, on average the statistical weight for method $\mathcal{M}_{1}, \mathcal{M}_{3}$ and $\mathcal{M}_{4}$ is multiplied by the same factor log $\alpha /(\alpha-1)$. For all these schemes splitting is thus expected to be a convenient population control tool, while it is ineffective for model $\mathcal{M}_{2}$. Variance reduction comes at the expense of an increased simulation time, because of the additional particle histories that must be simulated, which leads to a deterioration of the FOM. As $A$ increases, the average weight multiplier decreases, so that splitting becomes less efficient, as shown in Tab. 9. For $A=25$, splitting routines are never called during the simulation. 


\section{Conclusions}

In this work we have examined four different Monte Carlo models that can be used in order to formally solve the adjoint neutron transport equation. These models have been shown to belong to a broad class on Monte Carlo methods whereupon the simulation of fictitious particles ('adjunctons') obeying modified displacement and collision kernels allows estimating the adjoint neutron flux. Each scheme differs in the choice of adjuncton cross sections, scattering kernels and multiplicities, and otherwise shares the same simulation strategy.

In order to test the performances of the proposed schemes, we have considered a benchmark problem consisting in continuous-energy transport in infinite media starting from a mono-chromatic source, where reference solutions can be derived by solving a family of adjoint Placzek equations. Four kinds of nuclear reactions have been examined, namely, elastic and inelastic scattering, absorption and $(n, 2 n)$ multiplication. The relative standard deviations and the figures of merit of the Monte Carlo models have been computed for different physical configurations of interest.

For purely elastic scattering media, model $\mathcal{M}_{2}$ displays the highest figure of merit thanks to the introduction of a variance reduction scheme that is especially efficient when elastic scattering is the dominant reaction. The variance reduction method of model $\mathcal{M}_{4}$, although slightly less efficient than that of $\mathcal{M}_{2}$, yields a figure of merit larger than those of model $\mathcal{M}_{1}$ and $\mathcal{M}_{3}$. When inelastic scattering is taken into account, method $\mathcal{M}_{2}$ is very robust and displays the highest figure of merit and the smallest variance. If absorption is considered, the variance reduction technique of $\mathcal{M}_{2}$ becomes less efficient, and the figure of merit of this model becomes comparable to that of the other models when absorption is the dominant process. Yet, $\mathcal{M}_{2}$ has the prominent advantage of being universal, i.e., not specifically tailored for distinct collision kernels, and can be thus more easily implemented in existing production Monte Carlo codes.

The relevance of two population control techniques, namely Russian roulette and particle splitting, has been also shown, and their impact on the figures of merit of the Monte Carlo simulations has been discussed. Russian roulette prevents particle weights to decrease indefinitely, whereas splitting prevents particle weights to diverge indefinitely. Both techniques are greatly beneficial to the adjoint simulations.

It should be stressed that for the configurations tested here the maximum gain in the FOM is in most cases of the order of 10 at best: in this respect, the choice of an adjuncton model should be principally guided by a criterion of parsimony in view of a possible implementation in an existing Monte Carlo code. Based on these considerations, it would appear that the most judicious option would be to prefer model $\mathcal{M}_{1}$, because of its versatility, and switch to model $\mathcal{M}_{2}$ when needed. However, a wider panel of tests must be performed in order to assess the performances of adjuncton models for systems with coupled angle-energy scattering laws and with spatial leakages. Future work will then consist in extending the present analysis to other benchmark problems for finite-size media.

\section{A Constants for inelastic scattering}

We recall the definition of a few useful constants, as introduced in [13]. For model $\mathcal{M}_{1}$ and $\mathcal{M}_{2}$ let us define

$$
z_{\min }=\sqrt{1-\frac{\epsilon_{i}}{E_{i, \min }^{\prime}}}
$$

and

$$
z_{\max }=\sqrt{1-\frac{\epsilon_{i}}{E_{i, \max }^{\prime}}}
$$

If $E^{\prime} \geq \epsilon_{i}(A+1)^{2}, z_{\min }$ and $z_{\max }$ are obtained from the following formulae:

$$
z_{\text {min }}=\frac{-A+\sqrt{A^{2}+\left[A^{2}+(A+1)^{2} E^{\prime} / \epsilon_{i}\right]\left[(A+1)^{2} E^{\prime} / \epsilon_{i}-1\right]}}{A^{2}+(A+1)^{2} E^{\prime} / \epsilon_{i}}
$$

and

$$
z_{\max }=\frac{A+\sqrt{A^{2}+\left[A^{2}+(A+1)^{2} E^{\prime} / \epsilon_{i}\right]\left[(A+1)^{2} E^{\prime} / \epsilon_{i}-1\right]}}{A^{2}+(A+1)^{2} E^{\prime} / \epsilon_{i}} .
$$

Conversely, if $E^{\prime}<\epsilon_{i}(A+1)^{2}$, then $z_{\min }$ and $z_{\max }$ are obtained from

$$
z_{\text {min }}=\frac{A-\sqrt{A^{2}+\left[A^{2}+(A+1)^{2} E^{\prime} / \epsilon_{i}\right]\left[(A+1)^{2} E^{\prime} / \epsilon_{i}-1\right]}}{A^{2}+(A+1)^{2} E^{\prime} / \epsilon_{i}}
$$

and

$$
z_{\max }=\frac{A+\sqrt{A^{2}+\left[A^{2}+(A+1)^{2} E^{\prime} / \epsilon_{i}\right]\left[(A+1)^{2} E^{\prime} / \epsilon_{i}-1\right]}}{A^{2}+(A+1)^{2} E^{\prime} / \epsilon_{i}} .
$$


For model $\mathcal{M}_{4}$, we recall some definitions from [15]. The quantity $\bar{E}$ is defined as the arithmetic average energy between $E_{L}$ and $E_{R}$, namely,

$$
\bar{E}=\frac{1}{2}\left[E_{L}\left(E^{\prime}\right)+E_{R}\left(E^{\prime}\right)\right]
$$

where

$$
\begin{gathered}
E_{L}\left(E^{\prime}\right)=\frac{\epsilon_{i}}{1-y^{2}}, \\
E_{R}\left(E^{\prime}\right)=\frac{\epsilon_{i}}{1-\left(\frac{a}{b y}\right)^{2}} .
\end{gathered}
$$

The expressions for energies $E_{L}$ and $E_{R}$ are derived by assigning the coefficients

$$
\begin{array}{r}
a=\left[(A+1)^{2} E^{\prime} / \epsilon_{i}-1\right] / 2 A, \\
b=\left[(A+1)^{2} E^{\prime} / \epsilon_{i}+A^{2}\right] / 2 A, \\
y=2 a /[1+\sqrt{1+4 a b}] .
\end{array}
$$

\section{References}

1. I. Lux, L. Koblinger, Monte Carlo Particle Trasport Methods: Neutron and Photon Calculations (CRC Press, Boca Raton, Florida, 1991).

2. J. J. Duderstadt, W. R. Martin, Transport theory (Wiley, NY, 1979).

3. J. Lewins, Importance: the adjoint function (Pergamon press, London, 1965).

4. G. Goertzel, M. H. Kalos, Monte Carlo methods in transport problems, Prog. Nucl. Energy, 1, vol. 2 (Pergamon press, NY, 1958).

5. J. E. Hoogenboom, Nucl. Sci. Eng. 160, 1 (2008).

6. M. H. Kalos, Nucl. Sci. Eng. 33, 284 (1968).

7. L. B. Levitt, J. Spanier, Nucl. Sci. Eng. 37, 278 (1969).

8. B. Eriksson, C. Johansson, M. Leimdorfer, M. H. Kalos, Nucl. Sci. Eng. 37, 410 (1969).

9. C. W. Maynard, Nucl. Sci. Eng. 82, 97 (1961).

10. L. L. Carter, N. J. McCormick, Nucl. Sci. Eng. 39, 296 (1970).

11. D. C. Irving, Nucl. Eng. Design 15, 273 (1971).

12. A. De Matteis, Meccanica 3, 162 (1974).

13. J. E. Hoogenboom, Adjoint Monte Carlo methods in neutron transport calculations (PhD thesis, Delft, NL, 1977).

14. J. E. Hoogenboom, Nucl. Sci. Eng. 79, 357 (1981).

15. A. De Matteis, R. Simonini, Nucl. Sci. Eng. 65, 93 (1978).

16. L. L. Carter, Los Alamos scientific report LA4488 (1970).

17. R. J. Brissenden, Prog. Nucl. Energy 24, 129 (1990).

18. M. J. Grimstone, In Proceedings of the ANS Radiation Protection and Shielding Division Topical Conference (Nashville, April 19-23, 1998), pp. 143 - 150.

19. J. E. Hoogenboom, In Proceedings of the International Conference on Mathematics and Computation, Reactor Physics and Environmental Analysis in Nuclear Applications, M\&C 99 (Madrid, September 27-30, 1999), pp. 1912-1921.

20. J. E. Hoogenboom, Nucl. Sci. Eng. 143, 99 (2003).

21. C. M. Diop, O. Petit, C. Jouanne, M. Coste-Delclaux, Ann. Nucl. Energy 37 ,1186 (2010).

22. P. Saracco, S. Dulla, P. Ravetto, Eur. Phys. J. Plus 131, 412 (2016).

23. M. L. Williams, Nucl. Sci. Eng. 108, 355 (1991).

24. I. V. Serov, T. M. John, J. E. Hoogenboom, Nucl. Sci. Eng. 133, 55 (1999).

25. T. Ueki, J. E. Hoogenboom, J. L. Kloosterman, Nucl. Sci. Eng. 137, 117 (2001).

26. E. Brun, F. Damian, C. M. Diop, E. Dumonteil, F. X. Hugot, C. Jouanne, Y. K. Lee, F. Malvagi, A. Mazzolo, O. Petit,

J. C. Trama, T. Visonneau, A. Zoia, Ann. Nucl. Energy 82, 151 (2015).

27. G. Placzek, Phys. Rev. 69, 423 (1946).

28. V. Weisskopf, Phys. Rev. 52, 295 (1937). 